\title{
IMPULSIVE FRACTIONAL STOCHASTIC DIFFERENTIAL INCLUSIONS DRIVEN BY SUB-FRACTIONAL BROWNIAN MOTION WITH INFINITE DELAY AND SECTORIAL OPERATORS
}

\author{
MERYEM CHAOUCHE ${ }^{1, a}$ AND TOUFIK GUENDOUZI ${ }^{2, b}$
}

\footnotetext{
${ }^{1}$ Laboratory of Stochastic Models, Statistics and applications, University of Saida Dr Moulay Tahar P.O.Box 138 EN-Nasr, Saida 20000 Algeria.

${ }^{a}$ E-mail: meryem.chaouch@univ-saida.dz

${ }^{2}$ Laboratory of Stochastic Models, Statistics and applications, University of Saida Dr Moulay Tahar P.O.Box 138 EN-Nasr, Saida 20000 Algeria.

${ }^{b}$ E-mail: toufik.guendouzi@univ-saida.dz
}

\|\|

\section{Abstract}

We investigate the existence of mild solutions of impulsive fractional stochastic differential inclusions driven by sub-fractional Brownian motion $S_{Q}^{H}$ with infinite delay and non instantaneous impulses when the linear part is a fractional sectorial operators on separable Hilbert spaces. We consider the cases when the multivalued map is convex as well as non convex, a sufficient conditions for the existence are derived with the help of the multivalued fixed point theory and the measure of noncompactness.

\section{Introduction}

Differential equations and inclusions with fractional order arise in many engineering and scientific disciplines as the mathematical modeling of systems and processes in the fields of physics, mechanic, biology, ecology, aerodynamic, polymer rheology and many others. Fractional differential equations or inclusions also serve as an excellent tool for describing the memory and genetic properties of different materials and processes. As a consequence there was an intensive development of the theory of differential equations and

Received April 10, 2021.

AMS Subject Classification: 26A33, 34A37, 34A60, 34K40, 35R11, 60H15.

Key words and phrases: Mild solution, impulsive fractional stochastic differential inclusions, fractional Brownian motion, fractional sectorial operators, infinite delay, fractional derivative, fixed point. 
inclusions of fractional order. One can see the monographs of Abbas et al. [1], Kilbas et al. [22], Miller and Ross [27], Podlubny [23], Zhou [45], the survey of Agarwal et al [2] 3] and the references therein. Many articles have been devoted to the existence of solutions for fractional differential equations and inclusions, for example, [4] 6] [16] 28] [44]. As for the study of the existence of mild solutions for fractional differential inclusions, please see [13] [30] 39].

The theory of impulsive differential equations or inclusions has also attracted increasing attention because of its wide applicability in science and engineering. Impulsive differential inclusions arising from the real world problems to describe the dynamics of processes in which sudden, discontinuous jumps occurs. Such processes are naturally seen in biology, physics, medical fields, etc. Due to their significance, many authors have been established the solvability of impulsive differential inclusions. For the general theory and applications of such equations we refer the interested reader to Benchohra et al. 77, Graef et al. [17].

The deterministic systems often fluctuate due to noise, which is random or at least appears to be so. Therefor, we must move from deterministic problems to stochastic ones. As the generalization of classic impulsive differential and partial differential inclusions, impulsive stochastic differential and partial differential inclusions have attracted the researchers great interest, and some works have done on the existence results of mild solutions for these equation (see [24] 31] and references therein). Recently, attempts were made to combine fractional derivatives and stochastic differential inclusions. One can see [18] [19] [20] [41] and references therein.

On the other hand, fractional Brownian motion has become an object of intense study, due to its interesting properties and applications in various scientific areas including telecommunication, turbulence and finance. The fractional Brownian motion with Hurst parameter $H \in(0,1)$ is a suitable generalization of the classical Brownian motion, but exhibits lon-rang dependence, self similarity and which has stationary increments. When $H=\frac{1}{2}$ the $\mathrm{fBm}$ coincide with the classical Brownian motion. When $H \neq \frac{1}{2}$, the $\mathrm{fBm}$ is neither a semi-martingale nor a Markov process. For additional details on the fractional Brownian motion, we refer the reader to [25]. A general theory for the infinite dimensional stochastic differential equations driven by a fractional Brownian motion has begun to receive attention by various researchers see e.g., [12] 33]. The existence, uniqueness, stability and qualitative analysis 
of the mild solutions of stochastic differential equations driven by fractional Brownian motion with infinite delay have been studied by many authors (see 14] and references therein). Recently, Ren et al. 34 proved the existence and uniqueness of mild solution for a class of impulsive neutral stochastic functional integro-differential equations with infinite delay driven by standard cylindrical Wiener process and an independent cylindrical fractional Brownian motion with Hurst parameter $H \in\left(\frac{1}{2}, 1\right)$ in the Hilbert space. Boudaoui et al. [10] proved the existence of mild solutions to stochastic impulsive evolution equations with time delay, driven by fractional Brownian motion and Krasnoselski Schaefer type fixed point theorem. Ren et al. [35] proved the existence and uniqueness of the integral solution for a class of non-densely defined impulsive neutral stochastic functional differential equation driven by an independent cylindrical fractional Brownian motion with Hurst parameter $H \in\left(\frac{1}{2}, 1\right)$ in the Hilbert space. However, there are very few contributions regarding the existence of solutions to stochastic differential inclusions driven by fractional Brownian motion [11] 23]. An existence result of mild solutions for a first-order impulsive semi-linear stochastic functional differential inclusions driven by a fractional Brownian motion with infinite delay has been proved by Boudaoui et al. [11].

To the best of our Knowledge, there is no work reported on the impulsive fractional stochastic differential inclusions driven by sub-fractional Brownian motion with infinite delay and sectorial operators. Inspired by the previously mentioned works, in this article, we aim to study this interesting problem. We prove the existence of $\mathcal{P C}$ - mild solutions for impulsive fractional stochastic differential inclusions driven by sub-fractional Brownian motion with infinite delay and non-instantaneous impulses of the form

$$
\left\{\begin{array}{l}
{ }^{c} D_{t}^{\alpha} x(t) \in A x(t)+F\left(t, x_{t}\right)+g(t) \frac{d S_{Q}^{H}}{d t}, t \in\left(s_{i}, t_{i+1}\right], i=0,1, \ldots, N \\
x(0)=\varphi \in \mathcal{B}, \\
x(t)=I_{i}\left(t, x_{t}\right), t \in\left(t_{i}, s_{i}\right], i=1, \ldots, N
\end{array}\right.
$$

Where ${ }^{c} D^{\alpha}$ denotes the Caputo fractional derivative operator of order $\alpha \in$ $(0,1)$ with the lower limit zero; $x(\cdot)$ takes its values in the separable Hilbert spaces $\mathcal{H}$ with inner product $(\cdot, \cdot)_{\mathcal{H}}$ and norm $\|.\|_{\mathcal{H}} ; \mathrm{A}$ is a fractional sectorial operator defined on $\mathcal{H} ; F: J \times \mathcal{H} \rightarrow 2^{H}-\{\emptyset\}$ is a multifunction, $J:=[0, b]$, $0=t_{0}=s_{0}<t_{1} \leq s_{1} \leq s_{2} \leq t_{2}<\cdots<t_{N-1} \leq s_{N} \leq t_{N} \leq t_{N+1}=b$ 
be prefixed numbers; $g: J \rightarrow L_{Q}^{0}(\mathcal{K}, \mathcal{H}), \mathcal{K}$ is another real separable Hilbert space with inner product $(\cdot, \cdot)_{\mathcal{K}}$ and norm $\|\cdot\|_{\mathcal{K}}$. Here $L_{Q}^{0}(\mathcal{K}, \mathcal{H})$ denotes the space of all Q-Hilbert-Schmidt operators from $\mathcal{K}$ into $\mathcal{H}$ and $S_{Q}^{H}$ is an Q-sub-fBm with Hurst parameter $H \in\left(\frac{1}{2}, 1\right)$ which will be defined in the next section. The history $x_{t}:(-\infty, 0] \rightarrow \mathcal{H}, x_{t}(\theta)=x(t+\theta)$ belongs to some abstract phase $\mathcal{B}$ defined axiomatically in Section $2, I_{i} \in C\left(\left(t_{i}, s_{i}\right] \times \mathcal{B}, \mathcal{H}\right)$, for all $i=1, \ldots, N$. The initial data $\{\varphi(t):-\infty<t \leq 0\}$ is an $\mathcal{F}_{0}$-adapted $\mathcal{B}$-valued random variable independent of the sub-fBm with infinite second moment.

The outline of this paper is as follows. In the Section 2 we introduce some notations, definitions, preliminary facts about sub-fractional Brownian motion, the fractional calculus and an auxiliary lemma, which are used in the next sections. In Section 3, we give the existence of $\mathcal{P C}$-mild solution for (13) under both convexity and non-convexity conditions on the multi-valued right-hand side, and when the linear term $\mathrm{A}$ is fractional sectorial operator.

\section{Preliminaries}

In this section, we discuss some basic definitions, notations, theorems, lemmas and some basic facts about sub-fractional Brownian motion, the fractional calculus and sectorial operators.

Throughout this paper, the notations $\left(\mathcal{H},\|\cdot\|_{\mathcal{H}},(\cdot, \cdot)_{\mathcal{H}}\right)$ and $(\mathcal{K}, \| \cdot$ $\left.\|_{\mathcal{K}},(\cdot, \cdot)_{\mathcal{K}}\right)$ stand for the separable Hilbert spaces. The notation $\mathcal{C}(J, \mathcal{H})$ stand for the Banach space of continuous functions from $\mathrm{J}$ to $\mathcal{H}$ with supermum norm i.e., $\|x\|_{J}=\sup _{t \in J}\|x(t)\|$ and $L^{1}(J, \mathcal{H})$ denotes the Banach space of function $x: J \rightarrow \mathcal{H}$ which are Bochner integrable normed by $\|x\|_{L^{1}}=\int_{0}^{b}\|x(t)\| d t$, for all $x \in L^{1}(J, \mathcal{H})$. A measurable function $x: J \rightarrow \mathcal{H}$ is Bochner integrable if and only if $\|x\|$ is Lebesgue integrable. $B(\mathcal{H})$ is a Banach space of all linear bounded operator from $\mathcal{H}$ into itself with norm $\|F\|_{B(\mathcal{H})}=\sup \{\|F(x)\|:\|x\| \leq 1\}$.

Let $(\Omega, \mathcal{F}, \mathbb{P})$ be a complete probability space equipped with a normal filtration $\left\{\mathcal{F}_{t}\right\}_{t \geq 0}$ satisfying the usual conditions (i.e., right continuous and $\mathcal{F}_{0}$ containing all $\mathbb{P}$-null sets). 
Definition 1. The sub-fractional Brownian motion (sub-fBm in short) with Hurst parameter $H \in(0,1)$ is a mean zero Gaussian process $S^{H}=\left\{S_{t}^{H}: t \geq 0\right\}$ with $S_{H}^{0}=0$ and the covariance

$$
C_{H}(t, s)=\mathbb{E}\left[S_{t}^{H} S_{s}^{H}\right]=s^{2 H}+t^{2 H}-\frac{1}{2}\left[(s+t)^{2 H}+|t-s|^{2 H}\right]
$$

for all, $s, t \geq 0$.

For $H=\frac{1}{2}, S^{H}$ coincides with the standard Brownian motion B. $S^{H}$ is neither semimartingale nor a Markov process when $H \neq \frac{1}{2}$. The sub$\mathrm{fBm} S^{H}$ has properties analogous to those of fBm (self-similarity, long-range dependence, Holder paths), but it does not have stationary increments. More works for sub-fBm can be found in Bojdecki et al. [8] [9], Tudor [38], Shen et al. [37].

The sub-fractional Brownian motion satisfies the following estimates:

$$
\left[\left(2-2^{2 H-1}\right) \wedge 1\right]|t-s|^{2 H} \mathbb{E}\left|S^{H}(t)-S^{H}(s)\right|^{2} \leqslant\left[\left(2-2^{2 H-1}\right) \wedge 1\right]|t-s|^{2 H}
$$

Thus, Kolmogorov's continuity criterion implies that sub-fBm is holder continuous of order $\gamma$ for any $\gamma<H$ on any finite interval. Therefore, if $\mathrm{y}$ is a stochastic process with Holder continuous trajectories of order $\beta>1-H$ then the pathwise Riemann-Stieltjes integral $\int_{0}^{b} y_{t}(\omega) d S^{H}(t)(\omega)$ exists for all $b \geq 0$. In particular, if $H>\frac{1}{2}$, the pathwise integral $\int_{0}^{b} f^{\prime}\left(S_{t}^{H}\right) d S_{t}^{H}$ exists for all $f \in \mathcal{C}^{2}(\mathbb{R})$, and

$$
f\left(S_{b}^{H}\right)-f(0)=\int_{0}^{b} f^{\prime}\left(S_{t}^{H}\right) d S_{t}^{H}
$$

However, when $H<\frac{1}{2}$ the pathwise Riemann-Stieltjes integral $\int_{0}^{b} f^{\prime}\left(S_{t}^{H}\right) d S_{t}^{H}(\omega)$ does not exist. For more details, we refer the reader to [37] [42] [43].

Now we aim at introducing the Wiener integral with respect to one dimensional sub-fBm $S^{H}$. Fix a time interval $[0, b]$. We denote by $\Lambda$ the linear space of $\mathbb{R}$-valued step functions on $[0, b]$, that is, $y \in \Lambda$ if

$$
y(t)=\sum_{i=1}^{n-1} x_{i} 1_{\left[t_{i}, t_{i+1}\right](t)},
$$


Where $t \in[0, b], x_{i} \in \mathbb{R}$ and $0=t_{1}<t_{2}<\cdots<t_{n}=b$. For $y \in \Lambda$ we define its Wiener integral with respect to $S^{H}$ as

$$
\int_{0}^{b} y(s) d S_{Q}^{H}(s)=\sum_{i=1}^{n-1} x_{i}\left(S_{t_{i}+1}^{H}-S_{t_{i}}^{H}\right) .
$$

Let $\mathcal{H}_{\mathcal{S}^{H}}$ be the canonical Hilbert space associated to the sub-fBm $S^{H}$. That is $\mathcal{H}_{\mathcal{S}^{H}}$ is the cloture of the linear span $\Lambda$ with respect to the scalar product

$$
\left(1_{[0, t]}, 1_{[0, s]}\right)_{\mathcal{H}_{\mathcal{S}^{H}}}=C_{H}(t, s)
$$

We know that the covariance of sub-fBm can be written as

$$
\mathbb{E}\left[S_{t}^{H} S_{s}^{H}\right]=\int_{0}^{t} \int_{0}^{s} \eta_{H}(u, v) d u d v=C_{H}(t, s)
$$

where $\eta_{H}(u, v)=H(2 H-1)\left(|u-v|^{2 H-2}-(u+v)^{2 H-2}\right)$.

Equation (5) implies that

$$
(y, z)_{\mathcal{H}_{\mathcal{S}^{H}}}=\int_{0}^{t} \int_{0}^{s} y_{u} z_{v} \eta_{H}(u, v) d u d v
$$

for any pair step functions y and z on $[0, b]$. Consider the kernel

$$
K_{H}(t, s)=\frac{2^{1-H} \sqrt{\pi}}{\Gamma\left(H-\frac{1}{2}\right)} s^{3 / 2-H}\left(\int_{0}^{t}\left(x^{2}-s^{2}\right)^{H-3 / 2} d s\right) 1_{[0, t](s)}
$$

By Dzhaparidze and Van Zanten [15], we have

$$
C_{H}(t, s)=c_{H}^{2} \int_{0}^{t \wedge s} K_{H}(t, u) K_{H}(s, u) d u
$$

where

$$
c_{H}^{2}=\frac{\Gamma(1+2 H) \sin (\pi H)}{\pi} .
$$

Then, (8) implies that $C_{H}(s, t)$ is non-negative definite. Consider the linear operator $K_{H}^{*}: \Lambda \rightarrow L^{2}([0, b])$ defined by

$$
\left(K_{H}^{*} y\right)(s)=c_{H} \int_{s}^{r} y_{r} \frac{\partial K_{H}}{\partial r}(r, s) d r .
$$


Using (6) (8) we have

$$
\begin{aligned}
\left(K_{H}^{*} y, K_{H}^{*} z\right)_{L^{2}([0, b])} & =c_{H}^{2} \int_{0}^{b}\left(\int_{s}^{b} y_{r} \frac{\partial K_{H}}{\partial r}(r, s) d r\right)\left(\int_{s}^{b} z_{u} \frac{\partial K_{H}}{\partial u}(u, s) d u\right) d s \\
& =c_{H}^{2} \int_{0}^{b} \int_{0}^{b}\left(\int_{0}^{r \wedge u} \frac{\partial K_{H}}{\partial r}(r, s) \frac{\partial K_{H}}{\partial u}(u, s) d s\right) y_{r} z_{u} d r d u \\
& =c_{H}^{2} \int_{0}^{b} \int_{0}^{b} \frac{\partial^{2} K_{H}}{\partial r \partial u}(u, s) y_{r} z_{u} d r d u \\
& =H(2 H-1) \int_{0}^{b} \int_{0}^{b}\left(|u-r|^{2 H-2}-(u+r)^{2 H-2}\right) y_{r} z_{u} d r d u \\
& =(y, z)_{\mathcal{H}_{\mathcal{S}}} .
\end{aligned}
$$

As a consequence, the operator $K_{H}^{*}$ provides an isometry between the Hilbert space $\mathcal{H}_{\mathcal{S}^{H}}$ and $L^{2}([0, b])$. Hence, the process $\mathrm{W}$ defined by $W(t):=$ $S^{H}\left(\left(K_{H}^{*}\right)^{-1}\left(1_{[0, t]}\right)\right)$ is a Wiener process, and $S^{H}$ has the following Wiener integral representation:

$$
S^{H}(t)=c_{H} \int_{0}^{t} K_{H}(t, s) d W(s)
$$

because $\left(K_{H}^{*}\right)\left(1_{[0, t]}\right)(s)=c_{H} K_{H}(t, s)$. By [15], we have

$$
W(t)=\int_{0}^{t} Z_{H}(t, s) d S^{H}(s)
$$

where

$$
\begin{aligned}
Z_{H}(t, s)=\frac{s^{H-1 / 2}}{\Gamma(3 / 2-H)}[ & t^{H-3 / 2}\left(t^{2}-s^{2}\right)^{1 / 2-H} \\
& \left.-(H-3 / 2) \int_{s}^{t}\left(x^{2}-s^{2}\right)^{1 / 2-H} x^{H-3 / 2} d x\right]\left(1_{[0, t]}\right)(s) .
\end{aligned}
$$

In addition, for any $y \in \mathcal{H}_{\mathcal{S}^{H}}$,

$$
\int_{0}^{b} y(s) d S^{H}(s)=\int_{0}^{b}\left(K_{H}^{*} y\right)(t) d W(t)
$$

if and only if $K_{H}^{*} y \in L^{2}([0, b])$.

Also, denoting $L_{\mathcal{H}_{S} H}^{2}([0, b])=\left\{y \in \mathcal{H}_{S^{H}}, K_{H}^{*} y \in L^{2}([0, b])\right\}$. Since $H>\frac{1}{2}$, 
we have by (9) and Lemma 2.1 of [26],

$$
L^{2}([0, b]) \subset L^{\frac{1}{H}}([0, b]) \subset L_{\mathcal{H}_{S H}}^{2}([0, b]) .
$$

Lemma 1 ([29]). For $y \in L^{\frac{1}{H}}([0, b])$,

$$
H(2 H-1) \int_{0}^{b} \int_{0}^{b}\left|y_{r}\left\|y_{u}\right\| u-r\right|^{2 H-2} d r d u \leq C_{H}\|y\|_{L^{\frac{1}{H}([0, b])}},
$$

where $C_{H}=\left(\frac{H(2 H-1)}{\beta\left(2-2 H, H-\frac{1}{2}\right)}\right)^{1 / 2}$, with $\beta$ denoting the beta function.

Next, we are interested in considering a sub-fBm with values in Hilbert space and giving the definition of the corresponding stochastic integral.

Let $L(\mathcal{K}, \mathcal{H})$ denote the space of all bounded linear operators from $K$ into $H$ with the usual norm $\|\cdot\|_{L(\mathcal{K}, \mathcal{H})}$. Let $Q \in L(\mathcal{K}, \mathcal{H})$ be a non-negative self-adjoint operator. Denote by $L_{Q}^{0}(\mathcal{K}, \mathcal{H})$ the space of all $\xi \in L(\mathcal{K}, \mathcal{H})$ such that $\xi Q^{\frac{1}{2}}$ is a Hilbert-Schmidt operator. The norm is given by

$$
\|\xi\|_{L_{Q}^{0}(\mathcal{K}, \mathcal{H})}^{2}=\left\|\xi Q^{\frac{1}{2}}\right\|_{H S}^{2}=\operatorname{tr}\left(\xi Q \xi^{*}\right) .
$$

Then $\xi$ is called a Q-Hilbert-Schmidt operator from $\mathcal{K}$ to $\mathcal{H}$. Let $\left\{S_{n}^{H}(t)\right\}_{n \in \mathbb{N}}$ be a sequence of one-dimensionnal standard sub-fractional Brownian motions mutually independent on $(\Omega, \mathcal{F}, \mathbb{P})$. When one considers the following series:

$$
\sum_{n=1}^{\infty} S_{n}^{H}(t) e_{n}, \quad t \geq 0
$$

where $\left\{e_{n}\right\}_{n \in \mathbb{N}}$ is a complete orthonormal basis in $\mathcal{K}$ this series does not necessarily converge in the space $\mathcal{K}$. Thus we consider a $\mathcal{K}$-valued stochastic process $S_{Q}^{H}(t)$ given formally by the following series:

$$
S_{Q}^{H}(t)=\sum_{n=1}^{\infty} S_{n}^{H}(t) Q^{\frac{1}{2}} e_{n}, \quad t \geq 0 .
$$

If $\mathrm{Q}$ is a non-negative self-adjoint trace class operator, then this series converge in the space $\mathrm{K}$, that is, we have $S_{Q}^{H}(t) \in L^{2}(\Omega, K)$. Then above $S_{Q}^{H}(t)$ is well-defined as a $K$-valued Q-cylindrical sub-fractional Brownian motion 
with covariance operator $Q$. Let $y:[0, b] \longrightarrow L_{Q}^{0}(K, H)$ such that

$$
\sum_{n=1}^{\infty}\left\|K_{H}^{*}\left(y Q^{\frac{1}{2}} e_{n}\right)\right\|_{L^{2}([0, b] ; H)}<\infty
$$

Theorem 1. Let $y:[0, b] \longrightarrow L_{Q}^{0}(K, H)$ satisfy (11). Then its stochastic integral with respect to the sub-fBm $S_{Q}^{H}$ is defined, for $t \geq 0$, as follows

$$
\int_{0}^{t} y(s) d S_{Q}^{H}(s):=\sum_{n=1}^{\infty} \int_{0}^{t} y(s) Q^{\frac{1}{2}} e_{n} d S_{n}^{H}(s)=\sum_{n=1}^{\infty} \int_{0}^{t}\left(K^{*}\left(y Q^{\frac{1}{2}} e_{n}\right)\right) d W(s) .
$$

Notice that if

$$
\sum_{n=1}^{\infty}\left\|y(s) Q^{\frac{1}{2}} e_{n}\right\|_{L^{\frac{1}{H}([0, b] ; H)}}<\infty
$$

then in particular (11) holds, which follows immediately form (10).

Lemma 2 ([29]). For any $y:[0, b] \longrightarrow L_{Q}^{0}(K, H)$ such that (12) holds, and for any $u, v \in[0, b]$ with $u>v$,

$$
\mathbb{E}\left\|\int_{v}^{u} y(s) d S_{Q}^{H}(s)\right\|_{H}^{2} \leq C_{H}(u-v)^{2 H-1} \sum_{n=1}^{\infty} \int_{v}^{u}\left\|y(s) Q^{\frac{1}{2}} e_{n}\right\|_{H}^{2} d s .
$$

If, in addition,

$$
\sum_{n=1}^{\infty}\left\|y(s) Q^{\frac{1}{2}} e_{n}\right\|_{H}^{2} \quad \text { is uniformly convergent for } t \in[0, b],
$$

then

$$
\mathbb{E}\left\|\int_{v}^{u} y(s) d S_{Q}^{H}(s)\right\|_{H}^{2} \leq C_{H}(u-v)^{2 H-1} \int_{v}^{u}\|y(s)\|_{L_{Q}^{0}(K, H)}^{2} d s .
$$

In this paper, we suppose that $\mathcal{F}_{t}=\sigma\left\{S_{Q}^{H} ; 0 \leq s \leq t\right\}$ is the $\sigma$-algebra generated by the K-valued $Q$-cylindrical sub-fractional Brownian motion, $\mathcal{F}_{b}=\mathcal{F}$ and $L^{2}\left(\Omega, \mathcal{F}_{t}, \mathcal{H}\right)$ be the Hilbert spaces of all $\mathcal{F}_{t}$-adapted measurable square integrable random variables with values in $\mathcal{H}$. The notation $L_{2}^{\mathcal{F}}([0, b] ; \mathcal{H})$ stands for the Hilbert space of all square integrable and $\mathcal{F}_{t}$-measurable processes with the values in $\mathcal{H}$. 
Definition 2. The fractional integral of order $\alpha$ with the lower limit zero for a function $f$ is defined as

$$
I^{\alpha} f(t)=\frac{1}{\Gamma(\alpha)} \int_{0}^{t} \frac{f(s)}{(t-s)^{1-\alpha}} d s, \quad t>0, \alpha>0,
$$

provided the right-hand side is pointwise defined on $[0, \infty)$, where $\Gamma(\cdot)$ is the gamma function, which is defined by $\Gamma(\alpha)=\int_{0}^{\infty} t^{\alpha-1} e^{-t} d t$.

Definition 3. The Riemann-Liouville fractional derivative of order $\alpha>0$ $n-1<\alpha<n, n \in \mathbb{N}$, is defined as

$$
{ }^{(R-L)} D_{0+}^{\alpha} f(t)=\frac{1}{\Gamma(n-\alpha)}\left(\frac{d}{d t}\right)^{n} \int_{0}^{t}(t-s)^{n-1-\alpha} f(s) d s,
$$

where the function $f(t)$ has absolutely continuous derivative up to order $(n-1)$.

Definition 4. The Caputo derivative of order $\alpha>0$ for a function $f$ : $[0, \infty) \longrightarrow \mathbb{R}$ can be written as

$$
D^{\alpha} f(t)=D^{\alpha}\left(f(t)-\sum_{k=1}^{n-1} \frac{t^{k}}{k !} f^{k}(0)\right), \quad t>0, n-1<\alpha<n .
$$

\section{Remark 1.}

i. If $f(t) \in C^{n}[0, \infty)$, then

$$
{ }^{c} D^{\alpha} f(t)=\frac{1}{\Gamma(n-\alpha)} \int_{0}^{t} \frac{f^{(n)(s)}}{(t-s)^{\alpha+1-n}} d s=I^{n-\alpha} f^{n}(t), t>0, n-1<\alpha<n .
$$

ii. The Caputo derivative of a constant is equal to zero.

iii. If $f$ is an abstract function with values in $\mathcal{H}$, then integrals which appear in Definitions 2 and 3 are taken in Bochners sense.

We assume that the phase space $\left(\mathcal{B},\|\cdot\|_{\mathcal{B}}\right)$ is a seminoremed linear space of $\mathcal{F}_{0}$-measurable function mapping $(-\infty, 0]$ into $\mathcal{H}$, and satisfying the following fundamental axioms due to Hale and Kato [21].

i. If $x:(-\infty, b) \rightarrow \mathcal{H}, b>0$, is continuous on $(0, b]$ and $x_{0}$ in $\mathcal{B}$, then for every $t \in[0, a)$ the following conditions hold:

(a) $x_{t}$ is in $\mathcal{B}$; 
(b) $\|x(t)\|_{\beta} \leq \tilde{H}\left\|x_{t}\right\|_{\mathcal{B}}$;

(c) $\left\|x_{t}\right\|_{\mathcal{B}} \leq K(t) \sup \left\{\|x(s)\|_{\beta}: 0 \leq s \leq t\right\}+M(t)\left\|x_{0}\right\|_{\mathcal{B}}$, where $\tilde{H} \geq 0$ is a constant; $K, M:[0, \infty) \rightarrow[0, \infty), \mathrm{K}$ is continuous, $\mathrm{M}$ is locally bounded, and $\tilde{H}, \mathrm{~K}, \mathrm{M}$ are independent of $x(\cdot)$.

ii. For the function $x(\cdot)$ in i., $x_{t}$ is a $\mathcal{B}$-valued function $[0, a)$.

iii. The space $\mathcal{B}$ is complete.

The following result is a consequence of the phase space axioms.

Lemma $3([40])$. Let $x:(-\infty, b] \rightarrow \mathcal{H}$ be an $\mathcal{F}_{t}$-adapted measurable process such that the $\mathcal{F}_{0}$-adapted process $x_{0}=\varphi(t) \in L_{2}^{0}(\Omega, \mathcal{B})$ and the restriction $x: J \rightarrow L_{2}^{\mathcal{F}}(\Omega, \mathcal{B})$ is continuous, then

$$
\left\|x_{s}\right\|_{\mathcal{B}} \leq M_{b} \mathbb{E}\|\varphi\|_{\mathcal{B}}+K_{b} \sup _{0 \leq s \leq b} \mathbb{E}\|x(s)\|_{\mathcal{B}}
$$

where $K_{b}=\sup \{K(t): t \in J\}$ and $M_{b}=\sup \{M(t): t \in J\}$.

We introduce the space $\mathcal{P C}$ formed by all $\mathcal{F}_{t}$-adapted measurable square integrable $\mathcal{H}$-valued stochastic processes $\{x(t): t \in[0, b]\}$ such that $\mathrm{x}$ is continuous at $t \neq t_{i}, x\left(t_{t}\right)=x\left(t_{t}^{-}\right)$and $x\left(t_{t}^{+}\right)$exist for all $i=1, \ldots, N$. In this paper, we always assume that $\mathcal{P C}$ is endowed with the norm

$$
\|x\|_{\mathcal{P C}}=\left(\sup _{0 \leq t \leq b} \mathbb{E}\|x(t)\|^{2}\right)^{\frac{1}{2}} .
$$

Then $\left(\mathcal{P C},\|\cdot\|_{\mathcal{P C}}\right)$ is a Banach space.

Throughout this paper, we use the notation $\mathcal{P}(\mathcal{H})$ for the family of all nonempty subsets of $\mathcal{H}$. Let use introduce the following notations: $\mathcal{P}_{c l}(\mathcal{H})=\{\mathcal{Y} \in \mathcal{P}(\mathcal{H}): \mathcal{Y}$ is closed $\}, \mathcal{P}_{b d}(\mathcal{H})=\{\mathcal{Y} \in \mathcal{P}(\mathcal{H}): \mathcal{Y}$ is bounded $\}$, $\mathcal{P}_{c v}(\mathcal{H})=\{\mathcal{Y} \in \mathcal{P}(\mathcal{H}): \mathcal{Y}$ is convex $\}, \mathcal{P}_{c p}(\mathcal{H})=\{\mathcal{Y} \in \mathcal{P}(\mathcal{H}): \mathcal{Y}$ is compact $\}$, $\operatorname{conv}(\mathcal{Y})$ (respectively $\overline{\operatorname{conv}}(\mathcal{Y})$ ) be the convex hull (respectively, convex closed hull in $\mathcal{H}$ ) of a subset $\mathcal{Y}$ in $\mathcal{H}$.

A multi-valued map $\mathcal{G} \rightarrow \mathcal{P}(\mathcal{H})$ is convex (closed) valued if $\mathcal{G}(\mathcal{H})$ is convex (closed) for all $x \in \mathcal{H}$. $\mathcal{G}$ is bounded on bounded sets if $\mathcal{G}(B)=$ $\bigcup_{x \in B} \mathcal{G}(x)$ is bounded in $\mathcal{H}$ for any bounded set $B$ of $\mathcal{H}$, that is, $\sup _{x \in B}\left\{\sup \|y\|_{\mathcal{H}}\right.$ : $y \in \mathcal{G}(x)\}<\infty$. 
$\mathcal{G}$ is called upper semicountinuous (u.s.c) on $\mathcal{H}$ if, for each $x \in \mathcal{H}$, the set $\mathcal{G}(x)$ is nonempty closed subset of $\mathcal{H}$ and if, for each open set $V$ of $\mathcal{H}$ containing $\mathcal{G}(x)$, there exists an open neighborhood $N$ of $x$ such that $\mathcal{G}(N) \subseteq V$.

$\mathcal{G}$ is said to be completely continuous if $\mathcal{G}(B)$ is relatively compact, for every bounded subset $B$ of $\mathcal{H}$. If the multi-valued map $\mathcal{G}$ is completely countinuous with nonempty compact values, then $\mathcal{G}$ is u.s.c, if and only if $\mathcal{G}$ has closed graph i.e. $x_{n} \rightarrow x_{*}, y_{n} \rightarrow y_{*}, y_{n} \in \mathcal{G}\left(x_{n}\right)$ imply $y_{*} \in \mathcal{G}\left(x_{*}\right)$.

A multi-valued map $\mathcal{G}: J \rightarrow \mathcal{P}_{b d, c l, c v}(\mathcal{H})$ is measurable if for each $x \in \mathcal{H}$, the function $t \rightarrow D(x, \mathcal{G}(t))$ is measurable function on $\mathrm{J}$.

If $\mathcal{G}$ is a normed space, then the set $G_{\mathcal{G}}^{1}=\left\{f \in L^{1}([0, b], \mathcal{H}): f(t) \in\right.$ $\mathcal{G}(t)$, for a.e.t $\in[0, b]\}$ is called the set of selections of $\mathcal{G}$.

Definition $5([5])$. Let $\left\{\mathcal{Y}_{n}\right\}_{n \in \mathbb{N} \geq 1}$ be a sequence of subsets of $\mathcal{H}$. Suppose there is a compact and convex subset $\mathcal{Y} \subset \mathcal{H}$ such that for any neighborhood $\mathrm{N}$ of $\mathcal{Y}$ there is an $\mathrm{n}$ so that for any $m \geq n: \mathcal{Y}_{m} \subset N$. Then $\bigcap_{N>0} \overline{\operatorname{conv}}\left(\bigcup_{n \geq N} \mathcal{Y}_{n}\right) \subset \mathcal{Y}$.

Lemma $4([5])$. Every semicompact sequence in $L^{1}([0, b], \mathcal{H})$ is weakly compact in $L^{1}([0, b], \mathcal{H})$.

Now, we introduce the Hausdorff measure of noncompactness $\chi_{Z}(\cdot)$ defined by

$\chi_{Z}(B)=\inf \{\varepsilon>0: B$ admits a finite cover by balls of radius $\leq \varepsilon$ in $\mathcal{Z}\}$

for any Hilbert space $\mathcal{Z}$.

Some basic properties of $\chi_{\mathcal{Z}}(\cdot)$ are given in the following lemma.

Lemma 5. Let $Z$ be a real Hilbert space and $B$ be a bounded set in $Z$. Then, the following properties are satisfied:

i. $B$ is pre-compact if and only if $\chi_{Z}(B)=0$;

ii. $\chi_{Z}(B)=\chi_{Z}(\bar{B})=\chi_{Z}(\operatorname{conv} B)$, where $\bar{B}$ and conv $B$ are the closure and the convex hull of $B$, respectively;

iii. $\chi_{Z}(B) \leq \chi_{Z}(C)$ when $B \subseteq C$;

iv. $\chi_{Z}(B+C) \leq \chi_{Z}(B)+\chi_{Z}(C)$ where $B+C=\{x+y: x \in B, y \in C\}$;

v. $\chi_{Z}(B \cup C)=\max \left\{\chi_{Z}(B), \chi_{Z}(C)\right\}$; 
vi. $\chi_{Z}(\lambda B) \leq|\lambda| \chi_{Z}(B)$ for any $\lambda \in \mathbb{R}$;

vii. If the map $\phi: D(\phi) \subseteq \mathcal{Z} \rightarrow \mathcal{Z}^{\prime}$ is Lipschitz continuous with constant $k$ then $\chi_{Z}(\phi B) \leq k \chi_{Z}(B)$ for any bounded subset $B \subseteq D(\phi)$, where $\mathcal{Z}^{\prime}$ is another real Hilbert space;

viii. If $\left\{V_{n}\right\}_{n=1}^{\infty}$ is a decreasing sequence of bounded closed nonempty subset of $\mathbb{Z}$ and $\lim _{n \rightarrow \infty} \chi_{Z}\left(V_{n}\right)=0$, then $\bigcap_{n=1}^{\infty} V_{n}$ is nonempty and compact in $\mathcal{Z}$.

Lemma 6. Let $W$ be a closed convex subset of a Banach space $X$ and $R$ : $W \rightarrow P_{c k}(X)$ be a closed multifunction which is $\mathcal{X}$-condensing where $\mathcal{X}$ is a non singular measure of noncompactness defined on subsets of $W$, then $R$ has a fixed point.

Lemma 7. Let $W$ be a closed subset of a Banach space $X$ and $R: W \rightarrow$ $P_{k}(X)$ be a closed multifunction which is $\mathcal{X}$-condensing on every bounded subset of $W$, where $\mathcal{X}$ is a monotone measure of noncompactness defined on $X$. if the set of fixed points for $R$ is a bounded subset of $X$ then it is compact.

Lemma 8. Let $(X, d)$ be a complete metric space. If $R: X \rightarrow P_{c l b}(X)$ is contraction, then $R$ has a fixed point.

Lemma 9. Let $B$ be a bounded set in $Z$. Then for every $\varepsilon>0$ there is a sequence $\left(x_{n}\right)_{n \geq 1}$ in $B$ such that

$$
\chi(B) \leq 2 \chi\left\{x_{n}: n \geq 1\right\}+\varepsilon .
$$

Lemma 10. Let $\chi_{C(J, \mathcal{H})}$ be the Hausdorff measure of noncompactness on $C(J, \mathcal{H})$. If $W \subseteq C(J, \mathcal{H})$ is bounded, then for every $t \in J$,

$$
\chi(W(t)) \leq \chi_{C(J, \mathcal{H})}(W)
$$

where $W(t)=\{x(t): x \in W\}$. Furthermore, if $W$ is equicontinuous on $J$, Then the map $t \rightarrow \chi\{x(t): x \in W\}$ is continuous on $J$ and

$$
\chi_{C(J, \mathcal{H})}(W)=\sup _{t \in J} \chi\{x(t): x \in W\} .
$$

Lemma 11. Let $\left\{f_{n}: n \in \mathbb{N}\right\} \subset L^{p}(J, \mathcal{H}), p \geq 1$ be an integrable bounded sequence such that $\chi\left\{f_{n}: n \geq 1\right\} \leq \gamma(t)$, a.e.t $\in J$, where $\gamma \in L^{1}\left(J, \mathbb{R}^{+}\right)$. Then for each $\varepsilon>0$ there exists a compact $K_{\varepsilon} \subseteq E$, a measurable set $J_{\varepsilon} \subset J$, with measure less than $\varepsilon$, and a sequence of functions $\left\{g_{n}^{\varepsilon}\right\} \subseteq L^{p}(J, \mathcal{H}), t \in J$ and $\left\|f_{n}(t)-g_{n}^{\varepsilon}(t)\right\|<2 \gamma(t)+\varepsilon$, for every $n \geq 1$ and every $t \in J-J_{\varepsilon}$. 
Next, we are ready to recall some facts of fractional Cauchy problem. Bajlekova [46] studied the following linear fractional Cauchy problem

$$
\left\{\begin{array}{l}
D_{\alpha}^{c} x(t)=A x(t) \\
x(0)=x_{0} \in \mathcal{H}
\end{array}\right.
$$

where $\mathrm{A}$ is linear closed and $D(A)$ is dense.

Definition 6. A family $\left\{S_{\alpha}(t): t \geq 0\right\} \subset L(\mathcal{H})$ is called a solution operator for (13) if the following conditions are satisfied:

(a) $S_{\alpha}(t)$ is strongly continuous for $t \geq 0$ and $S_{\alpha}(t)=I$;

(b) $S_{\alpha}(t) D(A) \subset D(A)$ and $A S_{\alpha}(t) x=S_{\alpha}(t) A x$ for $x \in D(a)$ and $t \geq 0$;

(c) $S_{\alpha}(t) x$ is a solution of (13) for all $x \in D(A)$ and $t \geq 0$.

Definition 7. An operator A is said to be belong to $e^{\alpha}(M, \omega)$ if the solution operator $S_{\alpha}(\cdot)$ of (13) satisfies

$$
\left\|S_{\alpha}(t)\right\|_{L(\mathcal{H})} \leq M e^{\omega t}, t \geq 0
$$

for some constants $M \geq 1$ and $\omega \geq 0$.

Definition 8. A solution operator $S_{\alpha}(t)$ of (13) is called analytic if it admits an analytic extension to a sector $\Sigma_{\theta_{0}}=\left\{\lambda \in \mathbb{C}-\{0\}:\|\arg \lambda\|<\theta_{0}\right\}$ for some $\theta_{0} \in\left(0, \frac{\pi}{2}\right]$. An analytic solution operator is said to be of analyticity type $\left(\theta_{0}, \omega_{0}\right)$ if for each $\theta<\theta_{0}$ and $\omega>\omega_{0}$ there is an $M=M(\theta, \omega)$ such that

$$
\left\|S_{\alpha}(t)\right\|_{L(\mathcal{H})} \leq M e^{\omega \Re R t}, t \in \Sigma_{\theta} .
$$

Set

$$
\begin{gathered}
e^{\alpha}(\omega):=\bigcup\left\{e^{\alpha}(M, \omega): M \geq 1\right\} \text { and } e^{\alpha}:=\bigcup\left\{e^{\alpha}(\omega): \omega \geq 0\right\}, \\
A^{\alpha}\left(\theta_{0}, \omega_{0}\right)=\left\{A \in e^{\alpha}:\right. \text { A generates an analytic solution operator } \\
\left.S_{\alpha} \text { of type }\left(\theta_{0}, \omega_{0}\right)\right\} .
\end{gathered}
$$

Lemma 12. If $A \in A^{\alpha}\left(\theta_{0}, \omega_{0}\right)$ then

$$
\begin{aligned}
& \left\|S_{\alpha}(t)\right\|_{L(\mathcal{H})} \leq M e^{\omega t} \text { and }\left\|T_{\alpha}(t)\right\|_{L(\mathcal{H})} \leq C e^{\omega t}\left(1+t^{\alpha-1}\right) \\
& \text { for every } t>0, \omega>\omega_{0} \text {. So putting }
\end{aligned}
$$


We get

$$
\overline{M_{s}}:=\sup _{0 \leq t \leq b}\left\|S_{\alpha}(t)\right\|_{L(\mathcal{H})}, \overline{M_{T}}:=\sup _{0 \leq t \leq b} C e^{\omega t}\left(1+t^{1-\alpha}\right) .
$$

$$
\left\|S_{\alpha}(t)\right\|_{L(\mathcal{H})} \leq \overline{M_{s}},\left\|T_{\alpha}(t)\right\|_{L(\mathcal{H})} \leq t^{\alpha-1} \overline{M_{T}}
$$

Definition 9. Let $A \in A^{\alpha}\left(\theta_{0}, \omega_{0}\right)$ with $\theta_{0} \in\left(0, \frac{\pi}{2}\right]$ and $\omega_{0} \in \mathbb{R}$. A function $\mathrm{x}$ is called a mild solution of (1) if

$$
x(t)=\left\{\begin{array}{c}
S_{\alpha}(t) x_{0}+\int_{0}^{t} T_{\alpha}(t-s) f(s) d s+\int_{0}^{t} T_{\alpha}(t-s) g(s) d S_{Q}^{H}(s), t \in J_{0} \\
S_{\alpha}(t) x_{0}+S_{\alpha}\left(t-t_{1}\right) I_{1}\left(t_{1}^{-}\right)+\int_{0}^{t} T_{\alpha}(t-s) f(s) d s \\
+\int_{0}^{t} T_{\alpha}(t-s) g(s) d S_{Q}^{H}(s), t \in J_{1} \\
\vdots \quad N \quad x_{0}+\sum_{i=1}^{N} S_{\alpha}\left(t-t_{i}\right) I_{i}\left(x\left(t_{i}^{-}\right)\right)+\int_{0}^{t} T_{\alpha}(t-s) f(s) d s \\
+\int_{0}^{t} T_{\alpha}(t-s) g(s) d S_{Q}^{H}(s), t \in J_{N}
\end{array}\right.
$$

where $f \in S_{F(\cdot, x(\cdot))}$.

$S_{F(\cdot, x(\cdot)}$ is the set of the measurable selections of the multivalued map such that $S_{F(\cdot, x(\cdot))}=\left\{f \in L^{2}(J, \mathcal{H}): f(t) \in F(t, x(t))\right\}$.

\section{Existence of Mild Solution}

Theorem 2. Let $A \in A^{\alpha}\left(\theta_{0}, \omega_{0}\right)$ with $\theta \in\left(0, \frac{\pi}{2}\right]$ and $w_{0} \in \mathbb{R}, F: J \times \mathcal{H} \rightarrow$ $\mathcal{P}_{c v, c p}(\mathcal{H})$ a multifunction, $g: J \rightarrow L_{Q}^{0}(\mathcal{K}, \mathcal{H})$ and $I_{i} \in C\left(\left[t_{i}, s_{i}\right] \times \mathcal{B}, \mathcal{H}\right)$.

We assume the following conditions:

(H1) For any $x \in \mathcal{H}$, the multifunction $t \rightarrow F(t, x)$ is measurable and for all $t \in J, x \rightarrow F(t, x)$ is upper semicontinuous.

(H2) There exists a function $\varphi \in L^{\frac{1}{q}}\left(J, \mathbb{R}^{+}\right), q \in(0, \alpha)$ and a nondecreasing continuous function $\Theta: \mathbb{R}^{+} \rightarrow \mathbb{R}^{+}$such that for any $x \in \mathcal{H}$ $\|F(t, x)\| \leq \varphi(t) \Theta\|x\|$ 
(H3)

i) There exist a function $\beta \in L^{\frac{1}{q}}\left(J, \mathbb{R}^{+}\right), q \in(0, \alpha)$ satisfying

$$
4 \eta \overline{M_{T}}\|\beta\|_{L^{\frac{1}{q}\left(J, \mathbb{R}^{+}\right)}}<1
$$

where $\eta=\frac{b^{\alpha-q}}{\bar{w}^{1-q}}$ and $\bar{w}=\frac{\alpha-q}{1-q}$.

ii) For every bounded subset $Z \subseteq \mathcal{H}$

$\mathcal{X}(F(t, Z)) \leq \beta(t) \mathcal{X}(Z)$, for a.e. $t \in J$, where $\mathcal{X}$ is the Hausdorff measure of noncompactness in $\mathcal{H}$.

(H4) For $g:[0, b] \rightarrow L_{Q}^{0}(K, \mathcal{H})$ we assume the following conditions: for the complete orthonormal basis $\left\{e_{n}\right\}_{n \in \mathbb{N}}$ in $K$, we have:

$$
\begin{gathered}
\sum_{n=1}^{\infty}\left\|g Q^{\frac{1}{2}} e_{n}\right\|_{L^{2}([0, b], \mathcal{H})}<\infty \\
\sum_{n=1}^{\infty}\left\|g(t) Q^{\frac{1}{2}} e_{n}\right\|_{\mathcal{H}} \text { converges uniformly for } t \in[0, b] .
\end{gathered}
$$

(H5) The function $g: J \rightarrow L_{Q}^{0}(K, \mathcal{H})$ satisfies

$$
\int_{0}^{b}\|g(s)\|_{L_{Q}^{0}}^{2} d s=\Lambda<\infty
$$

(H6) For any $i=1,2, \ldots, N, I_{i}$ is continuous and there exists a positive constant $h_{i}$ such that

$$
\left\|I_{i}(t, x)\right\|^{2} \leq h_{i}\|x\|^{2}, x \in \mathcal{H}
$$

Then the problem (11) has a mild solution provided that there is $r>0$ such that

$$
\begin{aligned}
& 3{\overline{M_{s}}}^{2} e^{2 \omega R b} E\left\|x_{0}\right\|^{2}+\frac{3}{\alpha}{\overline{M_{T}}}^{2} b^{\alpha} \int_{0}^{b}(b-s)^{\frac{\alpha-1}{2}} E\|f(s)\|^{2} d s \\
& +3 c_{H} b^{2 H-1} \sum_{n=1}^{\infty} \int_{0}^{t}\left\|T_{\alpha}(b-s) Q^{\frac{1}{2} e_{n}}\right\|_{\mathcal{H}}^{2} d s \leq r .
\end{aligned}
$$

Proof. We transform the problem (1) into a fixed point problem, we define 
a multifunction $R: P C(J, \mathcal{H}) \rightarrow 2^{P C(J, \mathcal{H})}$ as follows:

For $x \in P C(J, \mathcal{H}), R(x)$ is the set of all functions $y \in R(x)$ such that

$$
y(t)=\left\{\begin{array}{c}
S_{\alpha}(t) x_{0}+\int_{0}^{t} T_{\alpha}(t-s) f(s) d s+\int_{0}^{t} T_{\alpha}(t-s) g(s) d S_{Q}^{H}(s), t \in J_{0} \\
S_{\alpha}(t) x_{0}+S_{\alpha}\left(t-t_{1}\right) I_{1}\left(t_{1}^{-}\right)+\int_{0}^{t} T_{\alpha}(t-s) f(s) d s \\
\quad+\int_{0}^{t} T_{\alpha}(t-s) g(s) d S_{Q}^{H}(s), t \in J_{1} \\
\vdots \quad \\
S_{\alpha}(t) x_{0}+\sum_{i=1}^{N} S_{\alpha}\left(t-t_{i}\right) I_{i}\left(x\left(t_{i}^{-}\right)\right)+\int_{0}^{t} T_{\alpha}(t-s) f(s) d s \\
\quad+\int_{0}^{t} T_{\alpha}(t-s) g(s) d S_{Q}^{H}(s), t \in J_{N}
\end{array}\right.
$$

where $f \in S_{F(\cdot, x(\cdot))}^{1}$. By the hypothesis (H1) the values of $\mathrm{R}$ are nonempty. It is clear that any fixed point for $\mathrm{R}$ is a mild solution for (1). so our aim is to show, by using Lemma 7 , that $\mathrm{R}$ has a fixed point. The proof will be given in the following steps.

Step 1. We proof that the values of $\mathrm{R}$ are closed.

Let $x \in P C(J, \mathcal{H})$ and $\left\{y_{n}: n \geq 1\right\}$ be a sequence in $R(x)$ which is convergent to y in $P C(J, \mathcal{H})$. Then according to the definition of $\mathrm{R}$, there is a sequence $\left\{f_{n}: n \geq 1\right\}$ in $S_{F(\cdot, x(\cdot))}^{1}$ such that for any $t \in J_{i}, i=0,1, \ldots, N$, we have

$$
y_{n}(t)=\left\{\begin{array}{c}
S_{\alpha}(t) x_{0}+\int_{0}^{t} T_{\alpha}(t-s) f_{n}(s) d s+\int_{0}^{t} T_{\alpha}(t-s) g(s) d S_{Q}^{H}(s), t \in J_{0} \\
S_{\alpha}(t) x_{0}+S_{\alpha}\left(t-t_{1}\right) I_{1}\left(t_{1}^{-}\right)+\int_{0}^{t} T_{\alpha}(t-s) f_{n}(s) d s \\
\quad+\int_{0}^{t} T_{\alpha}(t-s) g(s) d S_{Q}^{H}(s), t \in J_{1} \\
\vdots \quad \\
S_{\alpha}(t) x_{0}+\sum_{i=1}^{N} S_{\alpha}\left(t-t_{i}\right) I_{i}\left(x\left(t_{i}^{-}\right)\right)+\int_{0}^{t} T_{\alpha}(t-s) f_{n}(s) d s \\
\quad+\int_{0}^{t} T_{\alpha}(t-s) g(s) d S_{Q}^{H}(s), t \in J_{N}
\end{array}\right.
$$

By the assumption (H2) for every $n \geq 1$, and for a.e. $t \in J$ 


$$
\left\|f_{n}(t)\right\| \leq \varphi(t) \Theta(\|x\|) \leq \varphi(t) \Theta\left(\|x\|_{P C(J, \mathcal{H})}\right)
$$

This show that the set $\left\{f_{n}: n \geq 1\right\}$ is integrally bounded. Therefore for a.e. $t \in J\left\{f_{n}: n \geq 1\right\} \subset F(t, x(t))$, the set $\left\{f_{n}: n \geq 1\right\}$ is relatively compact in $\mathcal{H}$ for a.e.t $\in J$. Moreover, the set $\left\{f_{n}: n \geq 1\right\}$ is semicompact and then by Lemma 4 it is weakly compact in $L^{1}(J, \mathcal{H})$. So, without loss of generality we can assume that $f_{n}$ converges weakly to a function $f \in L^{1}(J, \mathcal{H})$. From Mazur's lemma, for any $j \in \mathbb{N}$ there exist a natural number $k_{0}(j)>j$ and a sequence of nonnegative real numbers $\lambda_{j, k}, k=j, \ldots, k_{0}(j)$ such that $\sum_{k=j}^{k_{0}(j)} \lambda_{j, k}=1$ and the sequence of convex combinations $z_{j}=\sum_{k=j}^{k_{0}(j)} \lambda_{j, k} f_{k}$, $j \geq 1$ converges strongly to $\mathrm{f}$ in $L^{1}(J, \mathcal{H})$ as $j \rightarrow \infty$. so we can suppose that $z_{j}(t) \rightarrow f(t)$ for a.e. $t \in J$. Since $\mathrm{F}$ takes convex and closed values, we obtain for a.e. $t \in J$

$$
f(t) \in \bigcap_{j \geq 1}\left\{z_{k}(t): k \geq j\right\} \subseteq \bigcap_{j \geq 1} \overline{\operatorname{conv}}\left\{f_{k}: k \geqslant j\right\} \subset F(t, x(t)) .
$$

Noting that, by (14) for every $t, s \in J, s \in[0, t]$ and $n \geq 1$

$$
\left\|T_{\alpha}(t-s) z_{n}(s)\right\| \leq(t-s)^{\alpha-1} \overline{M_{T}} \varphi(s) \Theta\|x\|_{P C(J, \mathcal{H})} .
$$

Next taking $\tilde{y}_{n}(t)=\sum_{k=j}^{k(j)} \lambda_{j k} y_{k},(19)$ implies

$$
\tilde{y}_{n}(t)=\left\{\begin{array}{c}
S_{\alpha}(t) x_{0}+\int_{0}^{t} T_{\alpha}(t-s) z_{n}(s) d s+\int_{0}^{t} T_{\alpha}(t-s) g(s) d S_{Q}^{H}(s), t \in J_{0} \\
S_{\alpha}(t) x_{0}+S_{\alpha}\left(t-t_{1}\right) I_{1}\left(t_{1}^{-}\right)+\int_{0}^{t} T_{\alpha}(t-s) z_{n}(s) d s \\
\quad+\int_{0}^{t} T_{\alpha}(t-s) g(s) d S_{Q}^{H}(s), t \in J_{1} \\
\vdots \quad \\
S_{\alpha}(t) x_{0}+\sum_{i=1}^{N} S_{\alpha}\left(t-t_{i}\right) I_{i}\left(x\left(t_{i}^{-}\right)\right)+\int_{0}^{t} T_{\alpha}(t-s) z_{n}(s) d s \\
\quad+\int_{0}^{t} T_{\alpha}(t-s) g(s) d S_{Q}^{H}(s), t \in J_{N}
\end{array}\right.
$$

But $\tilde{y}_{n}(t) \rightarrow y(t)$ and $\tilde{z}_{n}(t) \rightarrow f(t)$ for a.e. $t \in J$, therefore, by tending $\mathrm{n}$ to 
$\infty$ in (20), we get from the Lebesgue dominated convergence theorem that for every $i=0,1, \ldots, N$.

$$
y(t)=\left\{\begin{array}{c}
S_{\alpha}(t) x_{0}+\int_{0}^{t} T_{\alpha}(t-s) f(s) d s+\int_{0}^{t} T_{\alpha}(t-s) g(s) d S_{Q}^{H}(s), t \in J_{0} \\
S_{\alpha}(t) x_{0}+S_{\alpha}\left(t-t_{1}\right) I_{1}\left(t_{1}^{-}\right)+\int_{0}^{t} T_{\alpha}(t-s) f(s) d s \\
\quad+\int_{0}^{t} T_{\alpha}(t-s) g(s) d S_{Q}^{H}(s), t \in J_{1} \\
\vdots \\
S_{\alpha}(t) x_{0}+\sum_{i=1}^{N} S_{\alpha}\left(t-t_{i}\right) I_{i}\left(x\left(t_{i}^{-}\right)\right)+\int_{0}^{t} T_{\alpha}(t-s) f(s) d s \\
\quad+\int_{0}^{t} T_{\alpha}(t-s) g(s) d S_{Q}^{H}(s), t \in J_{N} .
\end{array}\right.
$$

This proves that $R(x)$ is closed.

Step 2. Set $B_{0}=\left\{x \in P C(J, \mathcal{H}):\|x\|_{P C} \leq r\right\}$. Obviously, $B_{0}$ is a bounded, closed and convex subset of $P C(J, \mathcal{H})$. We want to prove that $R\left(B_{0}\right) \subseteq B_{0}$. to show that, let $x \in B_{0}$ and $y \in R(x)$. By using (14), (17), (20); (H2) and Holder's inequality, we get for $t \in J_{0}$

$$
\begin{aligned}
E\|y(t)\|^{2}= & E\left\|S_{\alpha}(t) x_{0}+\int_{0}^{t} T_{\alpha}(t-s) f(s) d s+\int_{0}^{t} T_{\alpha}(t-s) g(s) d S_{Q}^{H}(s)\right\|^{2} \\
\leq & 3 E\left\|S_{\alpha}(t) x_{0}\right\|^{2}+3 E\left\|\int_{0}^{t} T_{\alpha}(t-s) f(s) d s\right\|^{2} \\
& +3 E\left\|\int_{0}^{t} T_{\alpha}(t-s) g(s) d S_{Q}^{H}(s)\right\|^{2} \\
\leq & 3 \bar{M}_{s}^{2} e^{2 \omega R t} E\left\|x_{0}\right\|^{2}+3 \bar{M}_{T}^{2} \frac{t^{\alpha}}{\alpha} \int_{0}^{t}(t-s)^{\frac{\alpha-1}{2}} E\|f(s)\|^{2} d s \\
& +3 c_{H} t^{2 H-1} \sum_{n=1}^{\infty} \int_{0}^{t}\left\|T_{\alpha}(t-s) Q^{\frac{1}{2}} e_{n}\right\|^{2} d s
\end{aligned}
$$

We get for every $t \in J_{i}, i=1,2, \ldots, N$

$$
\|y(t)\|_{P C}^{2} \leq r<\infty
$$

Therefore $R\left(B_{0}\right) \subseteq B_{0}$. 
Step 3. Let $Z=R\left(B_{0}\right)$. In this step we will show that the set defined as follows

$$
Z_{\overline{\mathrm{J}}_{i}}=\left\{y^{*} \in C\left(\bar{J}_{i}, \mathcal{H}\right): y^{*}(t)=y(t), t \in J_{i}, y^{*}\left(t_{i}\right)=y\left(t_{i}^{+}\right) y \in Z\right\}
$$

is equicontinuous for every $i=1,2, \ldots, N$.

Let $y \in Z$. Then there is $x \in B_{0}$ with $y \in R(x)$. According to the definition of $\mathrm{R}$, there is $f \in S_{F(\cdot, x(\cdot))}^{1}$ such that

$$
y(t)=\left\{\begin{array}{c}
S_{\alpha}(t) x_{0}+\int_{0}^{t} T_{\alpha}(t-s) f(s) d s+\int_{Q}^{t} T_{\alpha}(t-s) g(s) d S_{Q}^{H}(s), t \in J_{0} \\
S_{\alpha}(t) x_{0}+S_{\alpha}\left(t-t_{1}\right) I_{1}\left(t_{1}^{-}\right)+\int_{0}^{t} T_{\alpha}(t-s) f(s) d s \\
\quad+\int_{0}^{t} T_{\alpha}(t-s) g(s) d S_{Q}^{H}(s), t \in J_{1} \\
\vdots \quad \\
S_{\alpha}(t) x_{0}+\sum_{i=1}^{N} S_{\alpha}\left(t-t_{i}\right) I_{i}\left(x\left(t_{i}^{-}\right)\right)+\int_{0}^{t} T_{\alpha}(t-s) f(s) d s \\
\quad+\int_{0}^{t} T_{\alpha}(t-s) g(s) d S_{Q}^{H}(s), t \in J_{N} .
\end{array}\right.
$$

We consider the following cases:

Case 1 . When $i=0$, we consider two points t and $t+\delta$ be two points in $\bar{J}_{0}$, then:

$$
\begin{aligned}
\| & y^{*}(t+\delta)-y^{*}(t) \| \\
= & \| S_{\alpha}(t+\delta) x_{0}+\int_{0}^{t+\delta} T_{\alpha}(t+\delta-s) f(s) d s+\int_{0}^{t+\delta} T_{\alpha}(t+\delta-s) g(s) d S_{Q}^{H}(s) \\
& -S_{\alpha}(t) x_{0}-\int_{0}^{t} T_{\alpha}(t-s) f(s) d s-\int_{0}^{t} T_{\alpha}(t-s) g(s) d S_{Q}^{H}(s) \| \\
= & \|\left(S_{\alpha}(t+\delta)-S_{\alpha}(t)\right) x_{0}+\int_{0}^{t+\delta} T_{\alpha}(t+\delta-s) f(s) d s-\int_{0}^{t} T_{\alpha}(t-s) f(s) d s \\
& +\int_{0}^{t+\delta} T_{\alpha}(t+\delta-s) g(s) d S_{Q}^{H}(s)-\int_{0}^{t} T_{\alpha}(t-s) g(s) d S_{Q}^{H}(s) \| \\
= & \|\left(S_{\alpha}(t+\delta)-S_{\alpha}(t)\right) x_{0}+\int_{0}^{t}\left(T_{\alpha}(t+\delta-s)-T_{\alpha}(t-s)\right) f(s) d s
\end{aligned}
$$




$$
\begin{aligned}
& +\int_{t}^{t+\delta} T_{\alpha}(t+\delta-s) f(s) d s+\int_{0}^{t}\left(T_{\alpha}(t+\delta-s)-T_{\alpha}(t-s)\right) g(s) d S_{Q}^{H}(s) \\
& +\int_{t}^{t+\delta} T_{\alpha}(t+\delta-s) g(s) d S_{Q}^{H}(s) \| \\
\leq & \left\|\left(S_{\alpha}(t+\delta)-S_{\alpha}(t)\right) x_{0}\right\|+\left\|\int_{0}^{t}\left(T_{\alpha}(t+\delta-s)-T_{\alpha}(t-s)\right) f(s) d s\right\| \\
& +\left\|\int_{t}^{t+\delta} T_{\alpha}(t+\delta-s) f(s) d s\right\| \\
& +\left\|\int_{0}^{t}\left(T_{\alpha}(t+\delta-s)-T_{\alpha}(t-s)\right) g(s) d S_{Q}^{H}(s)\right\| \\
& +\left\|\int_{t}^{t+\delta} T_{\alpha}(t+\delta-s) g(s) d S_{Q}^{H}(s)\right\|, \\
E & \left\|y^{*}(t+\delta)-y^{*}(t)\right\|^{2} \\
\leq & 3 E\left\|\left(S_{\alpha}(t+\delta)-S_{\alpha}(t)\right) x_{0}\right\|^{2}+3 E\left\|\int_{0}^{t}\left(T_{\alpha}(t+\delta-s)-T_{\alpha}(t-s)\right) f(s) d s\right\|^{2} \\
& +3 E\left\|\int_{t}^{t+\delta} T_{\alpha}(t+\delta-s) f(s) d s\right\|^{2} \\
& +3 E\left\|\int_{0}^{t}\left(T_{\alpha}(t+\delta-s)-T_{\alpha}(t-s)\right) g(s) d S_{Q}^{H}(s)\right\|^{2} \\
& +3 E\left\|\int_{t}^{t+\delta} T_{\alpha}(t+\delta-s) g(s) d S_{Q}^{H}(s)\right\|^{2}:=3\left(G_{1}+G_{2}+G_{3}+G_{4}+G_{5}\right) .
\end{aligned}
$$

Where

$$
\begin{aligned}
& G_{1}=E\left\|\left(S_{\alpha}(t+\delta)-S_{\alpha}(t)\right) x_{0}\right\|^{2}, \\
& G_{2}=E\left\|\int_{0}^{t}\left(T_{\alpha}(t+\delta-s)-T_{\alpha}(t-s)\right) f(s) d s\right\|^{2}, \\
& G_{3}=E\left\|\int_{0}^{t}\left(T_{\alpha}(t+\delta-s)-T_{\alpha}(t-s)\right) g(s) d S_{Q}^{H}(s)\right\|^{2}, \\
& G_{4}=E\left\|\int_{t}^{t+\delta} T_{\alpha}(t+\delta-s) f(s) d s\right\|^{2}, \\
& G_{5}=E\left\|\int_{t}^{t+\delta} T_{\alpha}(t+\delta-s) g(s) d S_{Q}^{H}(s)\right\|^{2} .
\end{aligned}
$$

We only need to check $G_{i} \rightarrow 0$ as $\delta \rightarrow 0$ for every $i=1,2,3,4,5$. 
For $G_{1}$ we have

$$
\begin{aligned}
G_{1} & \left.=E \| S_{\alpha}(t+\delta)-S_{\alpha}(t)\right) x_{0} \|^{2} \\
& \leq\left\|S_{\alpha}(t+\delta)-S_{\alpha}(t)\right\|^{2} E\left\|x_{0}\right\|^{2} \\
& \leq\left\|S_{\alpha}(t+\delta)-S_{\alpha}(t)\right\|^{2} r^{\frac{1}{2}} .
\end{aligned}
$$

$\left.\sup _{0 \leq t \leq b} E \| S_{\alpha}(t+\delta)-S_{\alpha}(t)\right) x_{0}\left\|^{2} \leq \sup _{0 \leq t \leq b}\right\| S_{\alpha}(t+\delta)-S_{\alpha}(t) \| r^{\frac{1}{2}}$ $\left\|\left(S_{\alpha}(t+\delta)-S_{\alpha}(t)\right) x_{0}\right\|_{P C}^{2} \leq \sup _{0 \leq t \leq b}\left\|S_{\alpha}(t+\delta)-S_{\alpha}(t)\right\| r^{\frac{1}{2}}$

$$
\lim _{\delta \rightarrow 0}\left\|\left(S_{\alpha}(t+\delta)-S_{\alpha}(t)\right) x_{0}\right\|_{P C}^{2} \leq \lim _{\delta \rightarrow 0} \sup _{0 \leq t \leq b}\left\|S_{\alpha}(t+\delta)-S_{\alpha}(t)\right\| r^{\frac{1}{2}}=0 .
$$

uniformly for $x \in B_{0}$.

For $G_{2}$, we apply the Lebesgue dominated convergence theorem to get

$$
\begin{aligned}
G_{2} & =E\left\|\int_{0}^{t}\left(T_{\alpha}(t+\delta-s)-T_{\alpha}(t-s)\right) f(s) d s\right\|^{2} \\
& \leq E\left(\int_{0}^{t}\left\|\left(T_{\alpha}(t+\delta-s)-T_{\alpha}(t-s)\right)\right\|\|f(s)\| d s\right)^{2} \\
\sup _{0 \leq t \leq b} G_{2} & \leq \sup _{0 \leq t \leq b} E\left(\int_{0}^{t}\left\|\left(T_{\alpha}(t+\delta-s)-T_{\alpha}(t-s)\right)\right\|\|f(s)\| d s\right)^{2} \\
\lim _{\delta \rightarrow 0} \sup _{0 \leq t \leq b} G_{2} & \leq \lim _{\delta \rightarrow 0} \sup _{0 \leq t \leq b} E\left(\int_{0}^{t}\left\|\left(T_{\alpha}(t+\delta-s)-T_{\alpha}(t-s)\right)\right\|\|f(s)\| d s\right)^{2}=0 .
\end{aligned}
$$

For $G_{3}$ we use holder's inequality we obtain

$$
\begin{aligned}
& \left\|\int_{0}^{t}\left(T_{\alpha}(t+\delta-s)-T_{\alpha}(t-s)\right) g(s) d S_{Q}^{H}(s)\right\| \\
& =\left\|\int_{0}^{t} T_{\alpha}(t+\delta-s) g(s) d S_{Q}^{H}(s)-\int_{0}^{t} T_{\alpha}(t-s) g(s) d S_{Q}^{H}(s)\right\| \\
& \leq\left\|\int_{0}^{t} T_{\alpha}(t+\delta-s) g(s) d S_{Q}^{H}(s)\right\|+\left\|\int_{0}^{t} T_{\alpha}(t-s) g(s) d S_{Q}^{H}(s)\right\| \\
& \leq \sup _{0 \leq t \leq b} T_{\alpha}(t+\delta-s)\left\|\int_{0}^{t} g(s) d S_{Q}^{H}(s)\right\|+\sup _{0 \leq t \leq b} T_{\alpha}(t-s)\left\|\int_{0}^{t} g(s) d S_{Q}^{H}(s)\right\| \\
& \leq(t+\delta-s)^{\alpha-1} \bar{M}_{T}\left\|\int_{0}^{t} g(s) d S_{Q}^{H}(s)\right\|+\bar{M}^{T}(t-s)^{\alpha-1}\left\|\int_{0}^{t} g(s) d S_{Q}^{H}(s)\right\|
\end{aligned}
$$




$$
\begin{aligned}
& \left\|\int_{0}^{t}\left(T_{\alpha}(t+\delta-s)-T_{\alpha}(t-s)\right) g(s) d S_{Q}^{H}(s)\right\|^{2} \\
& \leq 2(t+\delta-s)^{2(\alpha-1)} \bar{M}_{T}^{2}\left\|\int_{0}^{t} g(s) d S_{Q}^{H}(s)\right\|^{2}+2 \bar{M}_{T}^{2}(t-s)^{2(\alpha-1)}\left\|\int_{0}^{t} g(s) d S_{Q}^{H}(s)\right\|^{2} \\
& E\left\|\int_{0}^{t}\left(T_{\alpha}(t+\delta-s)-T_{\alpha}(t-s)\right) g(s) d S_{Q}^{H}(s)\right\|^{2} \\
& \leq 2(t+\delta-s)^{2(\alpha-1)} \bar{M}_{T}^{2} E\left\|\int_{0}^{t} g(s) d S_{Q}^{H}(s)\right\|^{2} \\
& +2 \bar{M}_{T}^{2}(t-s)^{2(\alpha-1)} E\left\|\int_{0}^{t} g(s) d S_{Q}^{H}(s)\right\|^{2} \\
& \leq 2(t+\delta-s)^{2(\alpha-1)} \bar{M}_{T}^{2} c_{H} t^{2 H-1} \int_{0}^{t}\|g(s)\|_{L_{Q}^{0}(K, \mathcal{H})}^{2} d s \\
& +2 \bar{M}_{T}^{2}(t-s)^{2(\alpha-1)} c_{H} t^{2 H-1} \int_{0}^{t}\|g(s)\|_{L_{Q}^{0}(K, \mathcal{H})}^{2} d s \\
& \sup _{0 \leq t \leq b} E\left\|\int_{0}^{t}\left(T_{\alpha}(t+\delta-s)-T_{\alpha}(t-s)\right) g(s) d S_{Q}^{H}(s)\right\|^{2} \\
& \leq \sup _{0 \leq t \leq b}\left[2(t+\delta-s)^{2(\alpha-1)} \bar{M}_{T}^{2} c_{H} t^{2 H-1} \int_{0}^{t}\|g(s)\|_{L_{Q}^{0}(K, \mathcal{H})}^{2} d s\right. \\
& \left.+2 \bar{M}_{T}^{2}(t-s)^{2(\alpha-1)} c_{H} \int_{0}^{t}\|g(s)\|_{L_{Q}^{0}(K, \mathcal{H})}^{2} d s\right] \\
& \left(\sup _{0 \leq t \leq b} E\left\|\int_{0}^{t}\left(T_{\alpha}(t+\delta-s)-T_{\alpha}(t-s)\right) g(s) d S_{Q}^{H}(s)\right\|^{2}\right)^{\frac{1}{2}} \\
& \leq\left(\operatorname { s u p } _ { 0 \leq t \leq b } \left[2(t+\delta-s)^{2(\alpha-1)} \bar{M}_{T}^{2} c_{H} t^{2 H-1} \int_{0}^{t}\|g(s)\|_{L_{Q}^{0}(K, \mathcal{H})}^{2} d s\right.\right. \\
& \left.\left.+2 \bar{M}_{T}^{2}(t-s)^{2(\alpha-1)} c_{H} t^{2 H-1} \int_{0}^{t}\|g(s)\|_{L_{Q}^{0}(K, \mathcal{H})}^{2} d s\right]\right)^{\frac{1}{2}} . \\
& \lim _{\delta \rightarrow 0}\left(\sup _{0 \leq t \leq b} E\left\|\int_{0}^{t}\left(T_{\alpha}(t+\delta-s)-T_{\alpha}(t-s)\right) g(s) d S_{Q}^{H}(s)\right\|^{2}\right)^{\frac{1}{2}} \\
& \leq 2(t-s)^{\alpha-1} c_{H}^{\frac{1}{2}} t^{\frac{2 H-1}{2}}\left(\int_{0}^{t}\|g(s)\|_{L_{Q}^{0}(K, \mathcal{H})}^{2} d s\right)^{\frac{1}{2}} \\
& \left\|\int_{0}^{t}\left[T_{\alpha}(t+\delta-s)-T_{\alpha}(t-s)\right] g(s) d S_{Q}^{H}(s)\right\|_{P C}
\end{aligned}
$$




$$
\leq 2(t-s)^{\alpha-1} c_{H}^{\frac{1}{2}} t^{\frac{2 H-1}{2}}\left(\int_{0}^{t}\|g(s)\|_{L_{Q}^{0}(K, \mathcal{H})}^{2} d s\right)^{\frac{1}{2}} .
$$

For $G_{4}$, by the Holder's inequality we have

$$
\begin{aligned}
\| \int_{t}^{t+\delta} & T_{\alpha}(t+\delta-s) f(s) d s \| \\
& \leq \int_{t}^{t+\delta}\left\|T_{\alpha}(t+\delta-s)\right\|\|f(s)\| d s \\
& \leq \int_{t}^{t+\delta}(t+\delta-s)^{\alpha-1} \bar{M}_{T}\|f(s)\| d s \\
& \leq \bar{M}_{T} \int_{t}^{t+\delta}(t+\delta-s)^{\alpha-1}\|f(s)\| d s \\
& \leq \bar{M}_{T}\left(\int_{t}^{t+\delta}(t+\delta-s)^{(\alpha-1) p} d s\right)^{\frac{1}{p}}\left(\int_{t}^{t+\delta}\|f(s)\|^{q} d s\right)^{\frac{1}{q}} \\
& \leq \bar{M}_{T}\left(\int_{t}^{t+\delta}(t+\delta-s)^{\frac{(\alpha-1) q}{q-1}} d s\right)^{\frac{q-1}{q}}\left(\int_{t}^{t+\delta}\left\|\varphi^{q}(s)\right\| \Theta^{p}\|x\| d s\right)^{\frac{1}{p}} \\
& \leq \bar{M}_{T}\left(\int_{t}^{t+\delta}(t+\delta-s)^{\frac{\alpha-1}{q-1}} d s\right)^{q-1} \Theta\|x\|\left(\int_{t}^{t+\delta}\left\|\varphi^{q}(s)\right\| d s\right)^{\frac{1}{q}} \\
\leq & \bar{M}_{T}\left(\frac{\delta^{\omega}}{\omega}\right)^{q-1} \Theta\|x\|\|\varphi\| \varphi_{L^{\frac{1}{q}}\left(J, \mathbb{R}^{+}\right)} \cdot
\end{aligned}
$$

Where $\omega=\left(\frac{\alpha-1}{q-1}\right) q+1$

$$
\begin{aligned}
& \left\|\int_{t}^{t+\delta} T_{\alpha}(t+\delta-s) f(s) d s\right\| \leq \bar{M}_{T}\left(\frac{\delta^{\omega}}{\omega}\right)^{q-1} \Theta\|x\|\|\varphi\|_{L^{\frac{1}{q}\left(J, \mathbb{R}^{+}\right)}} \\
& \left\|\int_{t}^{t+\delta} T_{\alpha}(t+\delta-s) f(s) d s\right\|^{2} \leq \bar{M}_{T}^{2}\left(\frac{\delta^{\omega}}{\omega}\right)^{2(q-1)} \Theta^{2}\|x\|\|\varphi\|_{L^{\frac{1}{q}\left(J, \mathbb{R}^{+}\right)}}^{2} \\
& E\left\|\int_{t}^{t+\delta} T_{\alpha}(t+\delta-s) f(s) d s\right\|^{2} \leq \bar{M}_{T}^{2}\left(\frac{\delta^{\omega}}{\omega}\right)^{2(q-1)} E\left(\Theta^{2}\|x\|\|\varphi\|_{L^{\frac{1}{q}}\left(J, \mathbb{R}^{+}\right)}^{2}\right) \\
& \sup _{0 \leq t \leq b} E\left\|\int_{t}^{t+\delta} T_{\alpha}(t+\delta-s) f(s) d s\right\|^{2} \\
& \quad \leq \sup _{0 \leq t \leq b} \bar{M}_{T}^{2}\left(\frac{\delta^{\omega}}{\omega}\right)^{2(q-1)} E\left(\Theta^{2}\|x\|\|\varphi\|_{L^{\frac{1}{q}\left(J, \mathbb{R}^{+}\right)}}^{2}\right)
\end{aligned}
$$




$$
\begin{gathered}
\left(\sup _{0 \leq t \leq b} E\left\|\int_{t}^{t+\delta} T_{\alpha}(t+\delta-s) f(s) d s\right\|^{2}\right)^{\frac{1}{2}} \\
\quad \leq\left(\sup _{0 \leq t \leq b} \bar{M}_{T}^{2}\right)^{\frac{1}{2}}\left(\frac{\delta^{\omega}}{\omega}\right)^{q-1} E^{\frac{1}{2}}\left(\Theta^{2}\|x\|\|\varphi\|_{L^{\frac{1}{q}}\left(J, \mathbb{R}^{+}\right)}^{2}\right) \\
\lim _{\delta \rightarrow 0}\left(\sup _{0 \leq t \leq b} E\left\|\int_{t}^{t+\delta} T_{\alpha}(t+\delta-s) f(s) d s\right\|^{2}\right)^{\frac{1}{2}} \leq 0
\end{gathered}
$$

For $G_{5}$ we have

$$
\begin{aligned}
& \left\|\int_{t}^{t+\delta} T_{\alpha}(t+\delta-s) g(s) d S_{Q}^{H}(s)\right\| \leq \sup _{0 \leq t \leq b} T_{\alpha}(t+\delta-s)\left\|\int_{t}^{t+\delta} g(s) d S_{Q}^{H}(s)\right\| \\
& \left\|\int_{t}^{t+\delta} T_{\alpha}(t+\delta-s) g(s) d S_{Q}^{H}(s)\right\| \leq \sup _{0 \leq t \leq b} T_{\alpha}(t+\delta-s)\left\|\int_{t}^{t+\delta} g(s) d S_{Q}^{H}(s)\right\| \\
& \left\|\int_{t}^{t+\delta} T_{\alpha}(t+\delta-s) g(s) d S_{Q}^{H}(s)\right\|^{2} \\
& \leq\left(\sup _{0 \leq t \leq b} T_{\alpha}(t+\delta-s)\right)^{2}\left\|\int_{t}^{t+\delta} g(s) d S_{Q}^{H}(s)\right\|^{2} \\
& E\left\|\int_{t}^{t+\delta} T_{\alpha}(t+\delta-s) g(s) d S_{Q}^{H}(s)\right\|^{2} \\
& \leq\left(\sup _{0 \leq t \leq b} T_{\alpha}(t+\delta-s)\right)^{2} E\left\|\int_{t}^{t+\delta} g(s) d S_{Q}^{H}(s)\right\|^{2} \\
& \leq M^{2} 2 H(t+\delta-s)^{2 H-1} \int_{t}^{t+\delta}\|g(s)\|_{L_{Q}^{0}(K, \mathcal{H})}^{2} d s . \\
& \sup _{0 \leq t \leq b} E\left\|\int_{t}^{t+\delta} T_{\alpha}(t+\delta-s) g(s) d S_{Q}^{H}(s)\right\|^{2} \\
& \leq M^{2} 2 H \delta^{2 H-1} \int_{t}^{t+\delta}\|g(s)\|_{L_{Q}^{0}(K, \mathcal{H})}^{2} d s \\
& \left(\sup _{0 \leq t \leq b} E\left\|\int_{t}^{t+\delta} T_{\alpha}(t+\delta-s) g(s) d S_{Q}^{H}(s)\right\|^{2}\right)^{\frac{1}{2}} \\
& \leq M \sqrt{2 H} \delta^{\frac{2 H-1}{2}}\left(\int_{t}^{t+\delta}\|g(s)\|_{L_{Q}^{0}(K, \mathcal{H})}^{2} d s\right)^{\frac{1}{2}} .
\end{aligned}
$$




$$
\lim _{\delta \rightarrow 0}\left(\sup _{0 \leq t \leq b} E\left\|\int_{t}^{t+\delta} T_{\alpha}(t+\delta-s) g(s) d S_{Q}^{H}(s)\right\|^{2}\right)^{\frac{1}{2}} \leq 0 .
$$

Case 2. For $i \in\{1,2, \ldots, N\}$, let $t, t+\delta$ be two points in $J_{i}$. According to the definition of $\mathrm{R}$, we have

$$
\begin{aligned}
& \left\|y^{*}(t+\delta)-y^{*}(t)\right\|=\|y(t+\delta)-y(t)\| . \\
& \|y(t+\delta)-y(t)\|=\| S_{\alpha}(t+\delta) x_{0}+\sum_{i=1}^{N} S_{\alpha}\left(t+\delta-t_{i}\right) I_{i}\left(x\left(t_{i}^{-}\right)\right) \\
& +\int_{0}^{t+\delta} T_{\alpha}(t+\delta-s) f(s) d s+\int_{0}^{t+\delta} T_{\alpha}(t+\delta-s) g(s) d S_{Q}^{H}(s) \\
& -S_{\alpha}(t) x_{0}-\sum_{i=1}^{N} S_{\alpha}\left(t-t_{i}\right) I_{i}\left(x\left(t_{i}^{-}\right)\right) \\
& -\int_{0}^{t} T_{\alpha}(t-s) f(s) d s-\int_{0}^{t} T_{\alpha}(t-s) g(s) d S_{Q}^{H}(s) \| . \\
& \|y(t+\delta)-y(t)\| \leq\left\|\left(S_{\alpha}(t+\delta)-S_{\alpha}(t)\right) x_{0}\right\| \\
& +\sum_{k=1}^{i}\left\|S_{\alpha}\left(t+\delta-t_{k}\right) I_{k}\left(x\left(t_{k}^{-}\right)\right)-S_{\alpha}\left(t-t_{k}\right) I_{k}\left(x\left(t_{k}^{-}\right)\right)\right\| \\
& +\left\|\int_{0}^{t+\delta} T_{\alpha}(t+\delta-s) f(s) d s-\int_{0}^{t} T_{\alpha}(t-s) f(s) d s\right\| \\
& +\left\|\int_{0}^{t+\delta} T_{\alpha}(t+\delta-s) g(s) d S_{Q}^{H}(s)-\int_{0}^{t} T_{\alpha}(t-s) g(s) d S_{Q}^{H}(s)\right\| \\
& \|y(t+\delta)-y(t)\|^{2} \leq 3\left\|\left(S_{\alpha}(t+\delta)-S_{\alpha}(t)\right) x_{0}\right\|^{2} \\
& +3 \sum_{k=1}^{i}\left\|S_{\alpha}\left(t+\delta-t_{k}\right) I_{k}\left(x\left(t_{k}^{-}\right)\right)-S_{\alpha}\left(t-t_{k}\right) I_{k}\left(x\left(t_{k}^{-}\right)\right)\right\|^{2} \\
& +3\left\|\int_{0}^{t+\delta} T_{\alpha}(t+\delta-s) f(s) d s-\int_{0}^{t} T_{\alpha}(t-s) f(s) d s\right\|^{2} \\
& +3\left\|\int_{0}^{t+\delta} T_{\alpha}(t+\delta-s) g(s) d S_{Q}^{H}(s)-\int_{0}^{t} T_{\alpha}(t-s) g(s) d S_{Q}^{H}(s)\right\|^{2} . \\
& E\|y(t+\delta)-y(t)\|^{2} \leq 3 E\left\|\left(S_{\alpha}(t+\delta)-S_{\alpha}(t)\right) x_{0}\right\|^{2} \\
& +3 E \sum_{k=1}^{i}\left\|S_{\alpha}\left(t+\delta-t_{k}\right) J_{k}\left(x\left(t_{k}^{-}\right)\right)-S_{\alpha}\left(t-t_{k}\right) I_{k}\left(x\left(t_{k}^{-}\right)\right)\right\|^{2}
\end{aligned}
$$




$$
\begin{aligned}
& \quad+3 E\left\|\int_{0}^{t+\delta} T_{\alpha}(t+\delta-s) f(s) d s-\int_{0}^{t} T_{\alpha}(t-s) f(s) d s\right\|^{2} \\
& \quad+3 E\left\|\int_{0}^{t+\delta} T_{\alpha}(t+\delta-s) g(s) d S_{Q}^{H}(s)-\int_{0}^{t} T_{\alpha}(t-s) g(s) d S_{Q}^{H}(s)\right\|^{2} . \\
& \sup _{0 \leq t \leq b} E\|y(t+\delta)-y(t)\|^{2} \\
& \leq 3 \sup _{0 \leq t \leq b} E\left\|\left(S_{\alpha}(t+\delta)-S_{\alpha}(t)\right) x_{0}\right\|^{2} \\
& +3 \sup _{0 \leq t \leq b} E \sum_{k=1}^{i}\left\|S_{\alpha}\left(t+\delta-t_{k}\right) J_{k}\left(x\left(t_{k}^{-}\right)\right)-S_{\alpha}\left(t-t_{k}\right) I_{k}\left(x\left(t_{k}^{-}\right)\right)\right\|^{2} \\
& +3 \sup _{0 \leq t \leq b} E\left\|\int_{0}^{t+\delta} T_{\alpha}(t+\delta-s) f(s) d s-\int_{0}^{t} T_{\alpha}(t-s) f(s) d s\right\|^{2} \\
& +3 \sup _{0 \leq t \leq b} E\left\|\int_{0}^{t+\delta} T_{\alpha}(t+\delta-s) g(s) d S_{Q}^{H}(s)-\int_{0}^{t} T_{\alpha}(t-s) g(s) d S_{Q}^{H}(s)\right\|^{2} .
\end{aligned}
$$

As in the first case we get

$$
\lim _{\delta \rightarrow 0}\|y(t+\delta)-y(t)\|_{P C}=0
$$

Case 3. When $t=t_{i}, i=1,2, \ldots, N$, let $\lambda>0$ be such that $t_{i}+\lambda \in J_{i}$ and $\sigma>0$ such that $t_{i}<\sigma<t_{i}+\delta \leq t_{i+1}$, then we have

$$
\left\|y^{*}\left(t_{i}+\delta\right)-y^{*}\left(t_{i}\right)\right\|_{P C}=\lim _{\sigma \rightarrow t_{i}^{+}}\left\|y\left(t_{i}+\delta\right)-y(\sigma)\right\|_{P C} .
$$

According to the definition of $\mathrm{R}$ we get

$$
\begin{aligned}
& \left\|y\left(t_{i}+\delta\right)-y(\sigma)\right\|=\| S_{\alpha}\left(t_{i}+\delta\right) x_{0}+\sum_{k=1}^{N} S_{\alpha}\left(t_{i}+\delta-t_{k}\right) I_{k}\left(x\left(t_{k}^{-}\right)\right) \\
& +\int_{0}^{t_{i}+\delta} T_{\alpha}\left(t_{i}+\delta-s\right) f(s) d s+\int_{0}^{t_{i}+\delta} T_{\alpha}\left(t_{i}+\delta-s\right) g(s) d S_{Q}^{H}(s) \\
& -S_{\alpha}(\sigma) x_{0}-\sum_{k=1}^{N} S_{\alpha}\left(\sigma-t_{k}\right) I_{k}\left(x\left(t_{k}^{-}\right)\right)-\int_{0}^{\sigma} T_{\alpha}(\sigma-s) f(s) d s \\
& -\int_{0}^{t} T_{\alpha}(\sigma-s) g(s) d S_{Q}^{H}(s) \| .
\end{aligned}
$$




$$
\begin{aligned}
& \left\|y\left(t_{i}+\delta\right)-y(\sigma)\right\| \leq\left\|\left(S_{\alpha}\left(t_{i}+\delta\right)-S_{\alpha}(\sigma)\right) x_{0}\right\| \\
& \quad+\left\|\sum_{k=1}^{N}\left(S_{\alpha}\left(t_{i}+\delta-t_{k}\right)-S_{\alpha}\left(\sigma-t_{k}\right)\right) I_{k}\left(x\left(t_{k}^{-}\right)\right)\right\| \\
& \quad+\left\|\int_{0}^{t_{i}+\delta} T_{\alpha}\left(t_{i}+\delta-s\right) f(s) d s-\int_{0}^{\sigma} T_{\alpha}(\sigma-s) f(s) d s\right\| \\
& \quad+\left\|\int_{0}^{t_{i}+\delta} T_{\alpha}\left(t_{i}+\delta-s\right) g(s) d S_{Q}^{H}(s)-\int_{0}^{\sigma} T_{\alpha}(\sigma-s) g(s) d S_{Q}^{H}(s)\right\| .
\end{aligned}
$$

Arguing as in the first case we can see that

$$
\lim _{\delta \rightarrow 0, \sigma \rightarrow t_{i}^{+}}\left\|y\left(t_{i}+\delta\right)-y(\sigma)\right\|=0
$$

From the inequalities (23) - (24) we conclude that $Z_{\bar{I}_{i}}$ is equicontinuous for every $i=1,2, \ldots, m$.

Now for every $n \geq 1$, the set $B_{n}=\overline{c o n v} R\left(B_{n-1}\right)$. From step $1, B_{n}$ is a nonempty, closed and convex subset of $\mathcal{P C}(J, H)$. Moreover $B_{1}=$ $\overline{\operatorname{conv}} R\left(B_{0}\right) \subseteq B_{0}$. Also $B_{2}=\overline{\operatorname{conv}} R\left(B_{1}\right) \subseteq \overline{\operatorname{conv}} R\left(B_{0}\right) \subseteq B_{1}$ by induction the sequence $\left(B_{n}\right), n \geq 1$ is decreasing sequence of nonempty, closed and bounded subsets of $\mathcal{P C}(J, H)$.

We need only to show that the subset $B=\bigcap_{n=1}^{\infty} B_{n}$ is nonempty and compact in $\mathcal{P C}(J, H)$. by Lemma 5 , it is enough to show that

$$
\lim _{n \rightarrow \infty} \chi_{P C}\left(B_{n}\right)=0
$$

where $\chi_{P C}$ is the Hausdorff measure of noncompactness on $P C(J, H)$ defined in Section 2. In the next step we prove the equation (25).

Step 4. Let $n \geq 1$ be a fixed natural number and $\varepsilon>0$. In view of Lemma 9, there exists a sequence $\left(y_{k}\right)_{k \geq 1}$ in $R\left(B_{n-1}\right)$ such that

$$
\chi_{P C}\left(B_{n}\right)=\chi_{P C} R\left(B_{n-1}\right) \leq 2 \chi_{k}\left\{y_{k}: k \geq 1\right\}+\varepsilon .
$$

From the definition of $\chi_{P C}$, the above inequality becomes

$$
\chi_{P C}\left(B_{n}\right) \leq 2 \max _{i=0,1, \ldots, N} \chi_{i}\left(S_{\bar{J}_{i}}\right)+\varepsilon
$$


Where $S=\left\{y_{k}: k \geq 1\right\}$ and $\chi_{i}$ is the Hausdorff measure of noncompactness on $C\left(\bar{J}_{i}, H\right)$. As we have done in the previous step, we can show that $B_{n \mid \bar{J}_{i}}$, $i=0,1, \ldots, N$ is equicontinuous. Then, by applying Lemma 11 we get:

$$
\chi_{i}\left(S_{\bar{J}_{i}}\right)=\sup _{t \in \bar{J}_{i}} \chi(S(t))
$$

where $\chi$ is the Hausdorff measure of noncompactness on Z. Therefore, by using the nonsingularity of $\chi$, the inequality (26) becomes

$$
\begin{aligned}
\chi_{P C}\left(B_{n}\right) & \leq 2 \max _{i=0,1, \ldots, N}\left[\sup _{t \in \bar{J}_{i}} \chi(S(t))\right]+\varepsilon=2 \sup _{t \in J} \chi(S(t)) \\
& =2 \sup _{t \in J} \chi\left\{y_{k}(t): k \geq 1\right\}+\varepsilon .
\end{aligned}
$$

Now, since $y_{k} \in R\left(B_{n-1}\right), k \geq 1$ there exists $x_{k} \in B_{n-1}$ such that $y_{k} \in$ $R\left(x_{k}\right), k \geq 1$. By recalling the definition of $\mathrm{R}$ for every $k \geq 1$ there is $f_{k} \in S_{F\left(\cdot, x_{k}(\cdot)\right)}^{1}$ such that for every $t \in J$

$$
\chi\left\{y_{k}(t): k \geq 1\right\} \leq\left\{\begin{array}{c}
\chi\left\{S_{\alpha}(t) x_{0}\right\}+\chi\left\{\int_{0}^{t} T_{\alpha}(t-s) f_{k}(s) d s: k \geq 1\right\} \\
+\chi\left\{\int_{0}^{t} T_{\alpha}(t-s) g_{k}(s) d S_{Q}^{H}(s): k \geq 1\right\}, t \in J_{0} \\
\vdots \\
\chi\left\{S_{\alpha}(t) x_{0}\right\}+\sum_{p=1}^{N} \chi\left\{S_{\alpha}\left(t-t_{p}\right) I_{p}\left(x\left(t_{p}^{-}\right)\right): k \geqslant 1\right\} \\
+\chi\left\{\begin{array}{l}
t \\
\left.\int_{0}^{t} T_{\alpha}(t-s) f_{k}(s) d s: k \geq 1\right\} \\
+\chi\left\{\int_{0}^{t} T_{\alpha}(t-s) g_{k}(s) d S_{Q}^{H}(s): k \geq 1\right\}, t \in J_{N}
\end{array}\right.
\end{array}\right.
$$

Hence, for every $t \in J$ we have

$$
\chi\left\{S_{\alpha}(t) x_{0}: k \geq 1\right\}=0 .
$$

Moreover for every $p=1,2, \ldots, N$ and every $t \in J$

$$
\chi\left\{S_{\alpha}\left(t-t_{p}\right)\left(I_{p}\left(x_{k}\left(t_{p}^{-}\right)\right)\right): k \geq 1\right\}=0 .
$$

In order to be able to estimate

$$
\chi\left\{\int_{0}^{t} T_{\alpha}(t-s) f_{k}(s) d s: k \geq 1\right\}
$$


We can see that from (H3) it holds that for a.e. $t \in J$

$$
\begin{aligned}
\chi\left\{f_{k}(t): k \geq 1\right\} & \leq \chi\left\{F\left(t, x_{k}(t)\right): k \geq 1\right\} \\
& \leq \beta(t) \chi\left\{x_{k}(t): k \geq 1\right\} \\
& \leq \beta(t) \chi\left(B_{n-1}(t)\right) \\
& \leq \beta(t) \chi_{P C}\left(B_{n-1}(t)\right)=\gamma(t) .
\end{aligned}
$$

Furthermore, for any $k \geq 1$, by (H2), for almost $t \in J$, we have $\left\|f_{k}(t)\right\| \leq$ $\varphi(t) \Theta(r)$. Consequently, $f_{k} \in L^{\frac{1}{q}}(J, H), k \geq 1$. Note that $\gamma \in L^{\frac{1}{q}}\left(J, \mathbb{R}^{+}\right)$. Then from Lemma 11, there exists a compact set $K_{\varepsilon} \subseteq H$ and a measurable set $J_{\varepsilon} \subset J$. With a measure less than $\varepsilon$, and a sequence of functions $\left\{g_{k}^{\varepsilon}\right\} \subset$ $L^{\frac{1}{q}}(J, H)$ such that for every $s \in J,\left\{g_{k}^{\varepsilon}(s): k \geq 1\right\} \subseteq K_{\varepsilon}$, and $\| f_{k}(s)-$ $g_{k}^{\varepsilon}(s) \| \leq 2 \gamma(s)+\varepsilon$, for every $k \geq 1$ and every $s \in J_{\varepsilon}^{\prime}=J-J_{\varepsilon}$, then using Minkowski's inequality, we get

$$
\begin{aligned}
\left\|\int_{J_{\varepsilon}^{\prime}} T_{\alpha}(t-s)\left(f_{k}(s)-g_{k}^{\varepsilon}(s)\right) d s\right\| & \leq \bar{M}_{T} \eta\left[\int_{J_{\varepsilon}^{\prime}}(2 \gamma(s)+\varepsilon)^{\frac{1}{q}} d s\right]^{q} \\
& \leq \bar{M}_{T} \eta\|2 \gamma(s)+\varepsilon\|_{L^{\frac{1}{q}}\left(J, \mathbb{R}^{+}\right)} \\
& \leq \bar{M}_{T} \eta\left[\|2 \gamma(s)\|_{L^{\frac{1}{q}\left(J, \mathbb{R}^{+}\right)}}+\|\varepsilon\|_{L^{\frac{1}{q}}\left(J, \mathbb{R}^{+}\right)}\right] \\
& \leq \bar{M}_{T} \eta\left[\|2 \gamma(s)\|_{L^{\frac{1}{q}}\left(J, \mathbb{R}^{+}\right)}+2\|\varepsilon\|_{L^{\frac{1}{q}}\left(J, \mathbb{R}^{+}\right)}\right] \\
& \leq 2 \bar{M}_{T} \eta\left[\|\gamma(s)\|_{L^{\frac{1}{q}}\left(J, \mathbb{R}^{+}\right)}+\left(\int_{J} \varepsilon^{\frac{1}{q}} d s\right)^{q}\right] \\
& \leq 2 \bar{M}_{T} \eta\left[\|\gamma(s)\|_{L^{\frac{1}{q}}\left(J, \mathbb{R}^{+}\right)}+\varepsilon b^{q}\right] \\
& \leq 2 \bar{M}_{T} \eta\left[\left\|\beta \chi_{P C}\left(B_{n-1}\right)\right\|_{L^{\frac{1}{q}}\left(J, \mathbb{R}^{+}\right)}+\varepsilon b^{q}\right] \\
& \leq 2 \bar{M}_{T} \eta\left[\chi_{P C}\left(B_{n-1}\right)\|\beta\|_{L^{\frac{1}{q}}\left(J, \mathbb{R}^{+}\right)}+\varepsilon b^{q}\right] .
\end{aligned}
$$

Finally we get:

$$
\left\|\int_{J_{\varepsilon}^{\prime}} T_{\alpha}(t-s)\left(f_{k}(s)-g_{k}^{\varepsilon}(s)\right) d s\right\| \leq 2 \bar{M}_{T} \eta\left[\chi_{P C}\left(B_{n-1}\right)\|\beta\|_{L^{\frac{1}{q}\left(J, \mathbb{R}^{+}\right)}}+\varepsilon b^{q}\right]
$$


By Holder's inequality, we have:

$$
\begin{aligned}
\left\|\int_{J_{\varepsilon}} T_{\alpha}(t-s) f_{k}(s) d s\right\| & \leq \int_{J_{\varepsilon}}\left\|T_{\alpha}(t-s)\right\|\left\|f_{k}(s)\right\| d s \\
& \leq\left(\int_{J_{\varepsilon}}\left\|T_{\alpha}(t-s)\right\|^{\frac{1}{p}} d s\right)^{p}\left(\int_{J_{\varepsilon}}\left\|f_{k}(s)\right\|^{\frac{1}{q}} d s\right)^{q} \\
& \leq\left((t-s)^{\frac{\alpha}{p}}\right)^{p}\left(\bar{M}_{T}^{\frac{1}{p}}\right)^{p}\left(\int_{J_{\varepsilon}} d s\right)^{p}\left(\int_{J_{\varepsilon}}\left\|f_{k}(s)\right\|^{\frac{1}{q}} d s\right)^{q} \\
& \leq \eta \bar{M}_{T}\left(\int_{J_{\varepsilon}}(\Theta(r) \varphi(s))^{\frac{1}{q}} d s\right)^{q} \\
& \leq \eta \bar{M}_{T} \Theta(r)\left(\int_{J_{\varepsilon}} \varphi^{\frac{1}{q}}(s) d s\right)^{q} .
\end{aligned}
$$

Consequently we get

$$
\left\|\int_{J_{\varepsilon}} T_{\alpha}(t-s) f_{k}(s) d s\right\| \leq \eta \bar{M}_{T} \Theta(r)\left(\int_{J_{\varepsilon}} \varphi^{\frac{1}{q}}(s) d s\right)^{q}
$$

So by (31) (32), we derive

$$
\begin{aligned}
& \chi\left\{\int_{0}^{t} T_{\alpha}(t-s) f_{k}(s) d s: k \geq 1\right\} \\
& \leq \chi\left\{\int_{J_{\varepsilon}^{\prime}} T_{\alpha}(t-s) f_{k}(s) d s: k \geq 1\right\}+\chi\left\{\int_{J_{\varepsilon}} T_{\alpha}(t-s) f_{k}(s) d s: k \geq 1\right\} \\
& \leq \chi\left\{\int_{J_{\varepsilon}^{\prime}} T_{\alpha}(t-s)\left(f_{k}(s)-g_{k}^{\varepsilon}(s)\right) d s: k \geq 1\right\} \\
& +\chi\left\{\int_{J_{\varepsilon}^{\prime}} T_{\alpha}(t-s) g_{k}^{\varepsilon}(s) d s: k \geq 1\right\}+\chi\left\{\int_{J_{\varepsilon}} T_{\alpha}(t-s) f_{k}(s) d s: k \geq 1\right\} \\
& \leq 2 \bar{M}_{T} \eta\left[\chi_{P C}\left(B_{n-1}\right)\|\beta\| L^{\frac{1}{q}}\left(J, \mathbb{R}^{+}\right)\right. \\
& \left.\quad+\varepsilon b^{q}\right]+\eta \bar{M}_{T} \Theta(r)\left(\int_{J_{\varepsilon}} \varphi^{\frac{1}{q}}(s) d s\right)^{q} .
\end{aligned}
$$

By taking into account that $\varepsilon$ is arbitrary, we get for all $t \in J$

$$
\chi\left\{\int_{0}^{t} T_{\alpha}(t-s) f_{k}(s) d s: k \geq 1\right\} \leq 2 \bar{M}_{T} \eta \chi_{P C}\left(B_{n-1}\right)\|\beta\|_{L^{\frac{1}{q}}\left(J, \mathbb{R}^{+}\right)} .
$$


In order to estimate

$$
\chi\left\{\int_{0}^{t} T_{\alpha}(t-s) g_{k}(s) d S_{Q}^{H}: k \geq 1\right\} .
$$

We use Lemma 2 in order to calculate the following majoration:

$$
\begin{aligned}
& E\left\|\int_{0}^{t} T_{\alpha}(t-s) g_{k}(s) d S_{Q}^{H}(s)\right\|_{H}^{2} \leq c_{H} t^{2 H-1} \sum_{n=1}^{\infty} \int_{0}^{t}\left\|T_{\alpha}(t-s) g_{k}(s) Q^{\frac{1}{2}} e_{n}\right\|_{H}^{2} d s \\
& \sup _{0 \leq t \leq b} E\left\|\int_{0}^{t} T_{\alpha}(t-s) g_{k}(s) d S_{Q}^{H}(s)\right\|_{H}^{2} \\
& \quad \leq c_{H} t^{2 H-1} \sum_{n=1}^{\infty} \int_{0}^{b}\left\|T_{\alpha}(t-s) g_{k}(s) Q^{\frac{1}{2}} e_{n}\right\|_{H}^{2} d s \\
& \left\|\int_{0}^{t} T_{\alpha}(t-s) g_{k}(s) d S_{Q}^{H}(s)\right\|_{P C}^{2} \leq c_{H} t^{2 H-1} \sum_{n=1}^{\infty} \int_{0}^{b}\left\|T_{\alpha}(t-s) g_{k}(s) Q^{\frac{1}{2}} e_{n}\right\|_{H}^{2} d s .
\end{aligned}
$$

In an other hand we have:

$$
\begin{aligned}
\sum_{n=1}^{\infty} \int_{0}^{b}\left\|T_{\alpha}(t-s) g_{k}(s) Q^{\frac{1}{2}} e_{n}\right\|_{H}^{2} d s & \leq \sum_{n=1}^{\infty} \int_{0}^{b}\left\|T_{\alpha}(t-s)\right\|^{2}\left\|g_{k}(s) Q^{\frac{1}{2}} e_{n}\right\|_{H}^{2} d s \\
& \leq \sum_{n=1}^{\infty}\left\|g_{k}(s) Q^{\frac{1}{2}} e_{n}\right\|_{H}^{2} \int_{0}^{b}\left\|T_{\alpha}(t-s)\right\|^{2} d s \\
& \leq \bar{M}_{T}^{2} \frac{b^{2 \alpha-1}}{2 \alpha-1} \sum_{n=1}^{\infty}\left\|g_{k}(s) Q^{\frac{1}{2}} e_{n}\right\|_{H}^{2},
\end{aligned}
$$

and we know that

$$
\sum_{n=1}^{\infty}\left\|g_{k}(s) Q^{\frac{1}{2}} e_{n}\right\|_{H}^{2}<\infty
$$

So we have

$$
\left\|\int_{0}^{t} T_{\alpha}(t-s) g_{k}(s) d S_{Q}^{H}(s)\right\|_{P C} \leq c_{H} \frac{b^{2 H+2 \alpha-2}}{2 \alpha-1} \bar{M}_{T}^{2} K .
$$

where

$$
K=\sum_{n=1}^{\infty}\left\|g_{k}(s) Q^{\frac{1}{2}} e_{n}\right\|_{H}^{2}<\infty
$$


Then for every $t \in J$

$$
\begin{gathered}
\chi\left\{\int_{0}^{b} T_{\alpha}(t-s) g(s) d S_{Q}^{H}(s): k \geq 1\right\} \leq 0, \\
\chi\left\{y_{k}(t): k \geq 1\right\} \leq 2 \bar{M}_{T} \eta \chi_{P C}\left(B_{n-1}\right)\|\beta\|_{L^{\frac{1}{q}\left(J, \mathbb{R}^{+}\right)}} .
\end{gathered}
$$

The inequality (27) and the fact that $\varepsilon$ is arbitrary, imply

$$
\chi_{P C}\left(B_{n}\right) \leq 2\left[2 \bar{M}_{T} \eta \chi_{P C}\left(B_{n-1}\right)\|\beta\|_{L^{\frac{1}{q}\left(J, \mathbb{R}^{+}\right)}}\right] .
$$

By the previous steps $(1,2,3,4)$ we find that:

$$
0 \leq \chi_{P C}\left(B_{n}\right) \leq\left(4 \bar{M}_{T} \eta\|\beta\|_{L^{\frac{1}{q}\left(J, \mathbb{R}^{+}\right)}}\right)^{n-1} \chi_{P C}\left(B_{1}\right)
$$

Since this inequality is true for every $n \in \mathbb{N}$, by (16) and by tending $n \rightarrow \infty$, we obtain

$$
\lim _{n \rightarrow \infty} \chi_{P C}\left(B_{n}\right)=0
$$

Step 5. In this step, we will apply Lemma 5. The goal is to prove that the set $B=\bigcap_{n=1}^{\infty} B_{n}$ is a nonempty and compact subset of $P C(J, H)$. Moreover for every $B_{n}$ being bounded, closed and convex, B is also bounded closed and convex. Let us check that $R(B) \subseteq B$. Indeed, $R(B) \subseteq R\left(B_{n}\right) \subseteq$ $\overline{\operatorname{conv}} R\left(B_{n}\right)=B_{n+1}$.

For every $n \geq 1$, therefore $R(B) \subset \bigcap_{n=2}^{\infty} B_{n}$. On the other hand $B_{n} \subset B_{1}$ for every $n \geq 1$. So,

$$
R(B) \subset \bigcap_{n=2}^{\infty} B_{n}=\bigcap_{n=1}^{\infty} B_{n}=B
$$

Step 6 . In this step we show that the graph of the multi-valued function $R_{1 B}: B \rightarrow 2^{B}$ is closed. We consider a sequence $\left\{x_{n}\right\}_{n \geq 1}$ in $\mathcal{H}$ with $x_{n} \rightarrow x$ in $\mathcal{H}$ and let $y_{n} \in R\left(x_{n}\right)$ with $y_{n} \rightarrow y$ in $\operatorname{PC}(J, \mathcal{H})$. we will show that $y \in R(x)$. By recalling the definition of $\mathrm{R}$, there is $f_{n} \in S_{F\left(\cdot, x_{n}(\cdot)\right)}^{1}$ for any 
$n \geq 1$, such that

$$
y_{n}(t)=\left\{\begin{array}{l}
S_{\alpha}(t) x_{0}+\int_{0}^{t} T_{\alpha}(t-s) f_{n}(s) d s+\int_{0}^{t} T_{\alpha}(t-s) g(s) d S_{Q}^{H}(s), \quad t \in J_{0} \\
S_{\alpha}(t) x_{0}+S_{\alpha}\left(t-t_{1}\right) I_{1}\left(t_{1}^{-}\right)+\int_{0}^{t} T_{\alpha}(t-s) f(s) d s \\
+\int_{0}^{t} T_{\alpha}(t-s) g(s) d S_{Q}^{H}(s), \quad t \in J_{1} \\
\quad \vdots \\
S_{\alpha}(t) x_{0}+\sum_{k=0}^{i} S_{\alpha}\left(t-t_{k}\right) I_{k}\left(x\left(t_{k}^{-}\right)\right)+\int_{0}^{t} T_{\alpha}(t-s) f_{n}(s) d s \\
+\int_{0}^{t} T_{\alpha}(t-s) g(s) d S_{Q}^{H}(s), \quad t \in J_{i}, 1 \leq i \leq N
\end{array}\right.
$$

Observe that for every $n \geq 1$ and for a.e. $t \in J$

$$
\left\|f_{n}(t)\right\| \leq \varphi(t) \Theta\left(\left\|x_{n}(t)\right\|\right) \leq \varphi(t) \Theta(r)
$$

This show that the set $\left\{\left\{f_{n}: n \geq 1\right\}\right.$ is integrably bounded. In addition, the set $\left\{\left\{f_{n}(t): n \geq 1\right\}\right.$ is relatively compact for a.e. $t \in J$ by the assumption (H3) and the convergence of $\left\{x_{n}\right\}_{n \geq 1}$, imply that

$$
\chi\left\{f_{n}(t): n \geq 1\right\} \leq \chi\left\{F\left(t, x_{n}\right): n \geq 1\right\} \leq \beta(t) \chi\left\{x_{n}(t): n \geq 1\right\},
$$

then $\chi\left\{f_{n}(t): n \geq 1\right\}=0$.

So the sequence $\left.\left\{f_{n}\right\}_{n \geq 1}\right\}$ is semi-compact, hence by Lemma 4 it is weakly compact in $L^{1}(J, \mathcal{H})$. So without loss of generality we can assume that $f_{n}$ converges weakly to a function $L^{1}(J, \mathcal{H})$. From Mazur's lemma, for every $j \in \mathbb{N}$ there exist a natural number $k_{0}(j)>j$ and a sequence of nonnegative real numbers $\lambda_{j, k}, k=j, \ldots, k_{0}(j)$ such that $\sum_{k=j}^{k_{0}(j)} \lambda_{j, k}=1$ and the sequence of convex combinations $z_{j}=\sum_{k=j}^{k_{0}(j)} \lambda_{j, k} f_{k}, j \geq 1$ converges strongly to $\mathrm{f}$ in $L^{1}(J, \mathcal{H})$ as $j \rightarrow \infty$. So we may suppose that $z_{j}(t) \rightarrow f(t)$ for a.e.t $\in J$.

Let $t$ be such that $F(t, \cdot)$ is upper semicontinuous. Then, for any neighborhood $\mathrm{U}$ of $F(t, \cdot)$, there is a natural number $n_{0} \in \mathbb{N}$ so that for any $n \geq n_{0}$ we have $F\left(t, x_{n}(t)\right) \subseteq U$. 
Because the values of $\mathrm{F}$ are convex and compact, Definition 5 tells us that

$$
\bigcap_{j \geq 1} \overline{\operatorname{conv}}\left(\bigcup_{n \geq j} F\left(t, x_{n}(t)\right)\right) \subseteq F(t, x(t)) .
$$

As in step 1, from Mazur's theorem, there is a sequence $\left\{z_{n}: n \geq 1\right\}$ of convex combinations of $f_{n}$ such that for a.e.t $\in J$

$$
f(t) \in \bigcap_{j \geq 1} \overline{\left\{z_{n}(t): n \geq j\right\}} \subseteq \bigcap_{j \geq 1} \overline{\operatorname{conv}}\left\{\left\{f_{n}(t): n \geq j\right\}\right.
$$

and $z_{n}$ converges strongly to $f \in L^{1}(J, \mathcal{H})$. then, for a.e. $t \in J$

$$
\begin{aligned}
f(t) \in \bigcap_{j \geq 1} \overline{\left\{z_{n}(t): n \geq j\right\}} & \subseteq \bigcap_{j \geq 1} \overline{\operatorname{conv}}\left\{\left\{f_{n}(t): n \geq j\right\}\right. \\
& \subseteq \bigcap_{j \geq 1} \overline{\operatorname{conv}}\left(\bigcup_{n \geq j} F\left(t, x_{n}(t)\right)\right) \subseteq F(t, x(t)) .
\end{aligned}
$$

Then, by the continuity of $\mathrm{g}, S_{\alpha}, T_{\alpha}, I_{k}(k=1,2, \ldots, N)$ and by the same arguments used in step 1, we get from relation (33) that

$$
y(t)=\left\{\begin{array}{l}
S_{\alpha}(t) x_{0}+\int_{0}^{t}(t-s)^{\alpha-1} T_{\alpha}(t-s) f(s) d s \\
+\int_{0}^{t}(t-s)^{\alpha-1} T_{\alpha}(t-s) g(s) d S_{Q}^{H}(s), t \in J_{0} \\
\quad \vdots \\
S_{\alpha}(t) x_{0}+\sum_{k=0}^{i} S_{\alpha}\left(t-t_{k}\right) I_{k}\left(x\left(t_{k}^{-}\right)\right)+\int_{0}^{t}(t-s)^{\alpha-1} T_{\alpha}(t-s) f(s) d s \\
+\int_{0}^{t}(t-s)^{\alpha-1} T_{\alpha}(t-s) g(s) d S_{Q}^{H}(s), t \in J_{i}, 1 \leq i \leq N
\end{array}\right.
$$

Therefore, $y \in R(x)$. This show that the graph of $\mathrm{R}$ is closed.

As a result of the step 1-5 the multivalued $R_{1 B}: B \rightarrow 2^{B}$ is closed and $\chi_{P C^{-}}$-condensing, with nonempty convex compact values. By applying the fixed point theorem and Lemma 6 there exist $x \in B$ such that $x \in R(x)$. Then $\mathrm{x}$ is a PC-mild solution for the problem (11). 


\section{Example}

We consider the differential stochastic inclusion of the form

$$
\left\{\begin{array}{l}
{ }^{c} D_{t}^{\frac{1}{2}} y(t, z) \in \Delta y(t, z)+F\left(t, y_{t}\right)+g(t) \frac{d S_{Q}^{H}}{d t} \quad t \in[0,1], z \in[0, \pi] \\
y(t, 0)=y(t, \pi)=0, \\
y(\tau, z)=\varphi(\tau, z)(\tau, z) \in[0,1] \times[0, \pi] \\
y(t, z)=\int_{0}^{t} \eta_{i}(t-s) y(s, z) d s .
\end{array}\right.
$$

Where $\eta_{i}: \mathbb{R} \longrightarrow \mathbb{R}$ is continuous.

We take $\mathcal{H}=L^{2}[0,1]$ Hilbert spaces endowed with the norm $\|\cdot\|$ and $g: J \longrightarrow L_{Q}^{0}(\mathcal{H}, \mathcal{H})$, where $L_{Q}^{0}(\mathcal{H}, \mathcal{H})$ be the space of all operators Q Hilbert Schmidt.

Now we define the operator $A=\Delta$.

$$
D(A)=\left\{u \in C^{2+\lambda}[0, \pi]: u(0)=\pi \quad \text { and } \quad u(\pi)=0\right\}
$$

it is easy to see that the operator A is sectorial.

Now we suppose that $f_{i}:[0,1] \times \mathcal{H} \longrightarrow \mathcal{H}$

i $f_{1}, f_{2}$ are measurable and upper semi continuous.

ii $f_{1}, f_{2}$ are increasing functions

iii $f_{i}(t)<\varphi(t) \Theta\|x\|, \quad i=1,2$

Then we can transform the problem as follows

$$
\left\{\begin{array}{l}
{ }^{c} D_{t}^{\alpha} x(t) \in A x(t)+F\left(t, x_{t}\right)+g(t) \frac{d S_{Q}^{H}}{d t}, t \in\left(s_{i}, t_{i+1}\right], i=0,1, \ldots, N \\
x(0)=\varphi \in \mathcal{B}, \\
x(t)=I_{i}\left(t, x_{t}\right), t \in\left(t_{i}, s_{i}\right], i=1, \ldots, N .
\end{array}\right.
$$

From our assumptions on (i)-(ii) it follows that the multivalued function satisfy the conditions $\left(H_{1}\right)-\left(H_{2}\right)$.

All the assumptions in Theorem (3.1) are satisfied so our inclusion has a mild solution. 


\section{Conclusion}

In this paper we prove the existence of mild solution of impulsive fractional stochastic differential inclusion driven by Sub-fractional Brownian motion with infinite delay and sectorial operators. By using fixed point theory and Hausdorff measure of noncompactness, we investigated the stochastic differential inclusions, finally we have presented an example to illustrate the applicability of the new results.

\section{Acknowledgment}

This work was supported by the Thematic Agency of Research in Science and Technology ATRST- Algeria.

\section{References}

1. S. Abbas, M. Benchohra and G. M. N'Guérékata Topics in fractional Differential Equations, Springer, New York, ( 2012).

2. R. P. Agarwal, M. Benchohora and S. Hamani, Asurvey on existence results for bounday value problems of nonlinear fractional differential equations and inclusions, Act Appl. Math., 109 (2010), No.3, 973-1033.

3. R. P. Agarwal, M. Belmekki and M. Benchohora, Asurvey on semilinear differential equations and inclusions involving Riemann-Liouville fractional derivative, Adv. Differ. Equ., 2009, 981728 (2009).

4. B. Ahmad, R. P. Agarwal and A. Alsaedi, fractional differential equation and inclusions with semiperiodic and three-point boundary conditions, Boundary Value Problems, (20016), 2016:28.

5. J. P. Aubin and H. Frankoeska, Set-valued Analysis, Birkhuser, Boston, Basel, Berlin 1990.

6. M. Benchoh, J. Henderson, S. K. Ntouyas and A. Ouahab, Existence results for fractional order functional differential equations with infinite delay, J. Math. Anal. Appl., 338 (2008), No.2, 1340-1350.

7. M. Benchoh, J. Henderson, S. K. Ntouyas and A. Ouahab, Impulsive Differential Equations and Inclusions, Hindawi, Philadelphia, 2007.

8. T. L. G. Bojdecki, L. G. Gorostiza and A. Talarczyk, Some extensions of fractional Brownian motion and sub-fractional Brownian motion related to particle systems, Elect. Commun. Probab., 12 (2007), 161-172.

9. T. L. G. Bojdecki, L. G. Gorostiza and A. Talarczyk, Sub-fractional Brownian motion and its relation to occupation times, Stat. Prob. Lett., 69 (2004), 405-419. 
10. A. Boudaoui, T. Caraballo and A. Ouahab, Existence of mild solutions to stochastic delay evolution equations with a fractional Brownian motion and impulse, Stoch. Anal. Appl., 33 (2015), 244-258.

11. A. Boudaoui, T. Caraballo and A. Ouahab, Impulsive stochastic functional differential inclusion driven by a fractional Brownian motion with infinite delay, Math. Meth. Appl. Scien., 39 (2016), No.6, 1435-1451.

12. B. Boufoussi and S. Hajji, Functional differential equations driven by a fractional Brownian motion, Comput. Math. With Appl. Scien., 62 (2011), 746-754.

13. J. Cao, Y. Luo and G. Liu, Some results for impulsive fractional differential inclusions with infinite delay and sectorial operators in Banach spaces, Appl. Math. Comput., 273 (2016), 237-257.

14. T. Caraballo, M. J. Garrido-Atienza and T. Taniguchi, The existence and exponential behavior of solutions to stochastic delay evolution equations with a fractional Brownian motion, Nonl. Anal., 74 (2011), 3671-3684.

15. K. Dzhaparidze and H. Van Zanten, A series expansion of fractional Brownian motion, Probab. Theory Relat. Fields, 102 (2004), 39-5.5.

16. M. Feckan, Y. Zhou and J. Wang, On the concept and existence of solution for impulsive fractional differential equations, Commun. Nonlinear Sci. Numer. Simul., 17 (2012), No.7, 3050-3060.

17. J. R. Graef, J. Henderson and A. Ouahab, Impulsive differential inclusions: A fixed point Approach, De Gruyter Series in Nonlinear Analysis and Applications, 20 (2013).

18. T. Guendouzi and L. Bousmaha, Existence of solutions for fractional partial neutral stochastic function integro-differential inclusions with state-dependent delay and analytic resolvent operators, Vietnam J. Math., 43 (2015), No.4, 687-704.

19. T. Guendouzi and L. Bousmaha, Approximate controlability of fractional neutral stochastic functional integro-differential inclusions with infinite delay Qual, Theory Dyn. Syst., 13 (2014), No.1, 89-119.

20. T. Guendouzi and O. Benzatout, Existence of mild solutions for impulsive fractional stochastic differential inclusions with state-dependent delay, Ch. J. Math., V 2014 (2014), Article ID 981714, 13 pages.

21. J. K. Hale and J. Kato, Phase spaces for retarded equations with infinite delay, Funkc. Ekvacioj., 21 (1978), 11-41.

22. A. A. Kilbas, H. M. Srivastava and J. J. Trujillo, Theory and Applications of Fractional Differential Equations, Elsevier Science B.V, Amsterdam, 2006.

23. A. A. Levakov and M. M. Vas'kovskii, Existence of solutions of stochastic differential inclusions with standard and fractional Brownian motion, Diffe. Equa., 51 (2015), No.8, 991-997.

24. A. Lin and L. Hu, Existence results for impulsive neutral stochastic functional integro differential inclusions with non local initial conditions, Comput. Math. with Appl., 59 (2010), 64-73. 
25. B. B. Mandelbrot and J. W. V. Ness, Fractional Brownian motions, fractional noises and applications, SIAM. Rev., 10 (1968), 422-37.

26. I. Mendy, Parametric estimation for sub-fractional Ornstein- Uhlenbeck process, $J$. Stat. Plan. inference, 143 (2013), 633-674.

27. K. S. Miller and B. Ross, An Introduction to the Fractional Calculus and Fractional Differential Equations, John Wiley and Sons, New York. 1993.

28. S.K. Ntouyas, S. Etemad, On the existence of solutions for fractional differential inclusions with sum and integral boundary conditions, Appl. Math. Comput, 266 (2015), 235-243.

29. D. Nualart, The Malliavin Calculus and related Topics, 2nd edn. Springer, Berlin, 2006.

30. A. Ouahab, Fractional semilinear differential inclusions, Comput. Math. Appl., 64 (2012), 3235-3252.

31. J. Y. Park and J. U. Jeong, Existence results for impulsive neutral stochastic functional integro- differential inclusions with infinite delays, Adv. Differ. Eq., 2014: 17 (2014).

32. I. Podlubny, Fractional Differential Equations, Academic Press, San Diego, 1999.

33. Y. Ren, X. Cheng and R. Sakthivel On time-dependent stochastic evolution equations driven by fractional Brownian motion in Hilbert space with finite delay, Math. meth. Appl. Scien., 37 (2013), 2177-2184.

34. Y. Ren, X. Cheng and R. Sakthivel, Impulsive neutral stochastic functional integrodifferential equations with infinite delay driven by fBm, Appl. Math. Comput, 247 (2014), 205-212.

35. Y. Ren, T. Hou and R. Sakthivel, Non-densely defined impulsive neutral stochastic functional differential equations driven by $\mathrm{fBm}$ in Hilbert space with infinite delay, Front. Math. China, 10 (2015), No.2, 351-365.

36. Y. V. Rogovchenko, Nonlinear impulse evolution systems and applications to population models, J. Math. Anal. Appl., 207 (1997), 300-315.

37. G. Shen and C. Chen, Stochastic integration with respect to the sub-fractional Brownian with $\mathrm{H} \in\left(0, \frac{1}{2}\right)$, Stat. Prob. Letters, 82 (2012), 240-251.

38. C. Tudor, Some properties of the sub-fractional Brownian motion, Stochastics: An Intenational Jourmal of Probability and Stochastic Processes, 79 (2007), 431-448.

39. J. Wang, A.G. Ibrahim and M. Feckan, Nonlocal impulsive fractional differential inclusions with fractional sectorial operators on Banach spaces, Appl. Math. Comput, 257 (2015), 103-118.

40. Z. Yan and X. Yan, Existence of solutions for impulsive partial stochastic neutral integro differential equations with state-dependent delay, Collect. Math., 64 (2013), 235-250. 
"BN16N21" — 2021/7/14 — 22:17 — page 126 — \#40

41. Z. Yan and H. Zhang, Existence of solutions to impulsive fractional partial neutral stochastic integro-differential inclusions with state-dependent delay, Elec. Journal of Differ. Equa., 81 (2013), 1-21.

42. L. Yan, G. Shen and K. He, Ito's formula for a sub-fractional Brownian motion, Commun. Stoch. Anal., 5 (2011), 135-159.

43. L. C. Young, An inequality of the Holder type connected with Stieltjes integration, Acta Math., 67 (1936), 251-282.

44. Z. Zhang and B. Liu, Existence results of nondensely defined fractional evolution differential inclusions, J. Appl. Math., 2012, 316850 (2012).

45. Y. Zhou, Fractional Evolution Equations and Inclusions: Analyse and Control, San Diego, CA, Elsevier Science, 2016.

46. E. Bajlekova, Fractional evolution equations in Banach spaces (Ph.D. Thesis). Eindhoven University of Technology, 2011. 